\title{
Ectopic Expression of Homeobox Gene NKX2-1 in Diffuse Large B-Cell Lymphoma Is Mediated by Aberrant Chromatin Modifications
}

\author{
Stefan Nagel $^{1 *}$, Stefan Ehrentraut ${ }^{1}$, Jürgen Tomasch ${ }^{2}$, Hilmar Quentmeier ${ }^{1}$, Corinna Meyer ${ }^{1}$, \\ Maren Kaufmann', Hans G. Drexler', Roderick A. F. MacLeod'
}

1 Department of Human and Animal Cell Lines, Leibniz-Institute DSMZ-German Collection of Microorganisms and Cell Cultures, Braunschweig, Germany, 2 Microbial Communication, Helmholtz Centre for Infection Research, Braunschweig, Germany

\begin{abstract}
Homeobox genes encode transcription factors ubiquitously involved in basic developmental processes, deregulation of which promotes cell transformation in multiple cancers including hematopoietic malignancies. In particular, NKL-family homeobox genes TLX1, TLX3 and NKX2-5 are ectopically activated by chromosomal rearrangements in T-cell neoplasias. Here, using transcriptional microarray profiling and RQ-PCR we identified ectopic expression of NKL-family member NKX2-1, in a diffuse large B-cell lymphoma (DLBCL) cell line SU-DHL-5. Moreover, in silico analysis demonstrated NKX2-1 overexpression in 5\% of examined DLBCL patient samples. NKX2-1 is physiologically expressed in lung and thyroid tissues where it regulates differentiation. Chromosomal and genomic analyses excluded rearrangements at the NKX2-1 locus in SUDHL-5, implying alternative activation. Comparative expression profiling implicated several candidate genes in NKX2-1 regulation, variously encoding transcription factors, chromatin modifiers and signaling components. Accordingly, siRNAmediated knockdown and overexpression studies confirmed involvement of transcription factor HEY1, histone methyltransferase MLL and ubiquitinated histone H2B in NKX2-1 deregulation. Chromosomal aberrations targeting MLL at 11 q23 and the histone gene cluster HIST1 at 6 p22 which we observed in SU-DHL-5 may, therefore, represent fundamental mutations mediating an aberrant chromatin structure at NKX2-1. Taken together, we identified ectopic expression of NKX21 in DLBCL cells, representing the central player in an oncogenic regulative network compromising B-cell differentiation. Thus, our data extend the paradigm of NKL homeobox gene deregulation in lymphoid malignancies.
\end{abstract}

Citation: Nagel S, Ehrentraut S, Tomasch J, Quentmeier H, Meyer C, et al. (2013) Ectopic Expression of Homeobox Gene NKX2-1 in Diffuse Large B-Cell Lymphoma Is Mediated by Aberrant Chromatin Modifications. PLoS ONE 8(4): e61447. doi:10.1371/journal.pone.0061447

Editor: Yves St-Pierre, INRS, Canada

Received January 7, 2013; Accepted March 8, 2013; Published April 29, 2013

Copyright: (c) 2013 Nagel et al. This is an open-access article distributed under the terms of the Creative Commons Attribution License, which permits unrestricted use, distribution, and reproduction in any medium, provided the original author and source are credited.

Funding: The authors have no support or funding to report.

Competing Interests: The authors have declared that no competing interests exist.

* E-mail: sna@dsmz.de

\section{Introduction}

Lymphocytes originate from hematopoietic stem cells located in the bone marrow. While T-cells complete their development in the thymus, B-cells differentiate in various lymphoid tissues. Lymphoid malignancies emerge in the bone marrow or in secondary hematopoietic organs, acquiring both general and subtype specific mutations including chromosomal rearrangements. Accordingly, subtypes of the diffuse large B-cell lymphoma (DLBCL) differ in mutations and gene activities [1]. The sub-classification of this type of hematopoietic cancer represents a milestone in oncological research and has extensive implications for diagnosis and therapy. Two major subtypes, namely germinal center-derived B-cell and activated B-cell, are distinguished within the DLBCL entity [2]. It is believed that additional stratification should contribute to improved and better targeted therapies. Therefore, identification of novel genes or gene networks with diagnostic or therapeutic potential is of clinical interest.

Deregulated genes in leukemia/lymphoma comprise activated transcription factors $(\mathrm{TFs})$ and signaling components which are either physiologically expressed in early stages of hematopoietic development or ectopically induced. Notable examples include
TFs of the basic helix-loop-helix (bHLH) family or constituents of the NOTCH-signaling pathway [3]. The NOTCH gene itself may be activated by rare chromosomal translocations in $\mathrm{T}$-cell acute lymphoblastic leukemia/lymphoma (T-ALL) and by mutations affecting both T-ALL and B-cell malignancies. Targets of NOTCH-signaling comprise MYC and bHLH genes HES1 and HEY1 which may represent key oncogenes in malignant transformation [4].

Homeobox genes encode transcription factors frequently deregulated in cancers, including leukemia/lymphoma, impacting developmental processes during embryogenesis. According to their conserved homeobox sequences, this group of $\mathrm{TFs}$ has been classified into several subfamilies [5]. NKL family members regulate mesodermal differentiation and organogenesis [6], including NKX2-1 which regulates development of lung and thyroid, together with NKX2-5 and NKX3-1 which regulate that of the heart and prostate, respectively [7-10]. NKL-family members are involved in T-ALL [11], where activation usually follows chromosomal juxtaposition to potent transcriptional enhancers cognate to T-cell receptor genes at 7p14, 7q35 and $14 \mathrm{q} 11$, or the TF encoding gene BCL11B at 14q32 [12]. Exceptional, NKL family member NKX3-1 is ectopically 
expressed in T-ALL cells by the activating TFs TAL1, LYL1 and MSX2 rather than cytogenetically [13,14].

On the other hand the clustered HOX genes are usually activated by formation of aberrant chromatin structures in leukemia/lymphoma, although chromosomal aberrations are described in T-ALL [15]. Specific covalent modifications of core histones mediated by mutated MLL represent the most frequent mechanism of chromatin deregulation activating this homeobox gene group, including HOXA5 and HOXA10 [16]. MLL encodes a histone $\mathrm{H} 3$ methyltransferase and is associated with many cofactors in a ternary complex. Moreover, several genes encoding these cofactors are involved in fusion configurations with the MLL gene [17].

Here, we investigate aberrant expression of NKL homeobox gene NKX2-1 in B-cell lymphoma cell line SU-DHL-5. Our data expand the oncogenic role of NKL homeobox genes within the lymphoid system encompassing the B-cell lineage. We demonstrate mechanisms of NKX2-1 activation in addition to examining its downstream effects which include deregulation of cell differentiation in DLBCL.

\section{Materials and Methods}

\section{Cell lines and treatments}

Authenticated mycoplasma-free cell lines were drawn from the DSMZ Cell Lines Bank (Braunschweig, Germany) and cultivated as described previously [18]. Expression constructs and small interfering (si)RNAs were transfected into the cells by electroporation using the EPI-2500 impulse generator (Fischer, Heidelberg, Germany) at $350 \mathrm{~V}$ for $10 \mathrm{~ms}$ and subsequently incubated for 16 h. Expression constructs for HEY1 and HES1 were obtained from Origene (Wiesbaden, Germany). SiRNA oligonucleotides and AllStars negative Control siRNA (termed here siCTR) were obtained from Qiagen (Hilden, Germany). Cell stimulations were performed by treatment with recombinant human Bone morphogenetic protein (BMP) 4, Interleukin (IL)4, IL10, Transforming growth factor beta (TGFb), Tumor necrosis factor alpha (TNFa), or WNT5B for $16 \mathrm{~h}$ at concentrations of $20 \mathrm{ng} / \mathrm{ml}$ (R \& D Systems, Wiesbaden, Germany). Treatments with $\gamma$-secretase inhibitor N-[N-(3,5-Difluorophenacetyl)-L-alanyl]-S-phenylglycine t-butyl ester (DAPT) (Sigma, Taufkirchen, Germany) were performed at concentrations of $1 \mu \mathrm{M}$ for $16 \mathrm{~h}$, with cAMPderivate (8-4-Chlorophenylthio-adenosine $3^{\prime}, 5^{\prime}$-cyclic monophosphate, Sigma) and cGMP-derivate (8-bromoguanosine 3' $3^{\prime}$ '-cyclic monophosphate, Sigma) at concentrations of $100 \mu \mathrm{M}$, with Sildenafil (Tocris Bioscience, Bristol, UK) at concentrations of $100 \mu \mathrm{M}$ and with the NFkB-inhibitor (Calbiochem, Darmstadt, Germany) as indicated in the text. Treatments with anti-TGF beta-1 (clone 9016.2, Thermo Scientific, Germany) were performed with $1 \mu \mathrm{g} / \mathrm{ml}$ for $16 \mathrm{~h}$. DZNep was kindly provided by Dr. Marquez (NIH, Frederick, MD, USA) and applied as described recently [19].

\section{Expression profiling}

For quantification of gene expression via profiling, we used data obtained by gene chips HG U133 Plus 2.0 from Affymetrix (High Wycombe, UK). The datasets were generated at the University of Würzburg and generously provided by Prof. Andreas Rosenwald (Institute of Pathology, University of Würzburg, Germany) or obtained from the National Center for Biotechnology Information (NCBI) Gene Expression Omnibus (GEO) (www.ncbi.nlm.nih. gov/gds) or from the National Cancer Institute (NCI). The NGI microarray data for SU-DHL-5 are available through the accession numbers SU-DHL-5_SS392729_HG-
U133_Plus_2_HCHP-201545_.CEL, SU-DHL-5 _SS392730_HG-U133_Plus_2_HCHP-201546_.CEL, and SUDHL-5_SS392731_HG-U133_Plus_2_HCHP-201547_.CEL

which were combined in this study. Analyses of expression data were performed using Microsoft Excel and online programs. For creation of heat maps we used CLUSTER version 2.11 and TREEVIEW version 1.60 (http://rana.lbl.gov/EisenSoftware. htm). Expression data of 203 DLBCL patient samples were obtained from the NCBI GEO database (accession number GSE11318) as published recently [20]. Statistical analyses of NKX2-1 (dataset 211024_s_at) expression were performed using R-software.

\section{Genomic array analysis}

Genome-wide copy number analysis was performed using the Affymetrix Genotyping Console GTC Software version 4.0 (Affymetrix) and visualized by the Affymetrix GTC-Browser program. The 500K-array dataset for SU-DHL-5 was obtained from the National Cancer Institute (Bethesda, MD, USA), GSK Cancer Cell Line Genomic Profiling Data (https://cabig.nci.nih. gov/community/caArray_GSKdata/).

\section{Chromosomal analyses}

Fluorescent in-situ hybridization (FISH) and spectral karyotyping (SKY) analyses were performed as described previously [21,22]. RP11-BAC-clones were obtained from BacPac Resources, Childrens Hospital Oakland Research Institute (CA, USA), prepared using the Big BAC DNA Kit (Princeton Separations, Adelphia, NJ, USA) and directly labelled by nick translation with dUTP-fluors (Dyomics, Jena, Germany). For analysis of the NKX2-1 locus BACs RP11-676A19, RP11-945C4, and RP1174D5 were labelled with Dy-590, Dy-547 and Dy-495, respectively. For analysis of the HEY1 locus we used RP11-300M12 (Dy590), RP11-89I14 (Dy-547) and RP11-24P11 (Dy-495), for that of MLL we used RP1 1-347D24 (Dy-547), RP1 1-91A14 (Dy-590) and RP11-770K18 (Dy-495), and for HIST1 RP11-958P15 (Dy-495), RP11-846O7 (Dy-547) and RP11-6N6 (Dy-590). Fluorescence images were captured and analyzed with an Axio-Imager microscope (Zeiss, Göttingen, Germany) configured to a dual Spectral Imaging FISH and SKY system (Applied Spectral Imaging, Neckarhausen,Germany).

\section{Polymerase chain-reaction (PCR) analyses}

Total RNA from cell lines was extracted using TRIzol reagent (Invitrogen, Karlsruhe, Germany). Total human RNA isolated from peripheral blood mononuclear cells (PBMC), bone marrow (BM), lymph nodes (LN), thymus, lung and thyroid was obtained from Clontech (Saint-Germain-en-Laye, France), and RNA isolated from CD3-positive T-cells and CD19-positive B-cells from Miltany Biotec (Bergisch Gladbach, Germany). cDNA was subsequently synthesized from $5 \mu \mathrm{g}$ RNA by random priming, using Superscript II (Invitrogen).

Real-time quantitative expression analysis (RQ-PCR) was performed by the 7500 Fast Real-time System, using commercial buffer and primer sets (Applied Biosystems, Darmstadt, Germany). For normalization of expression levels we used TATA box binding protein (TBP). Quantitative analyses were performed in triplicate and repeated twice. The standard deviations are indicated in the figures as bars.

Reverse transcription (RT) PCR was performed using taqpol (Qiagen), oligonucleotides as listed in Table S1 (MWG Eurofins, Martinsried, Germany) and the thermocycler TGradient (Biometra, Göttingen, Germany). 


\section{Protein analysis}

Sodium dodecyl sulphate polyacrylamid gel electrophoresis (SDS-PAGE) was performed in the Mini-Protean 3 system (BioRad, München, Germany). Gels were either stained with PageBlue (Fermentas, Vilnius, Lithuania) or processed for Western blot analysis via the semi-dry method. Proteins obtained from cell lysates were transferred onto nitrocellulose membranes (Bio-Rad) and blocked with $5 \%$ dry milk powder dissolved in phosphatebuffered-saline buffer (PBS). The following antibodies were used: anti-NKX2-1 (EP1584Y, Abgent, Heidelberg, Germany), antiNKX3.1 (clone 3-9, Invitrogen), anti-ERK1 (K-23, Santa Cruz Biotechnology, Heidelberg, Germany). The secondary antibodies were labelled with peroxidase and detected by Western LightningECL (Perkin Elmer, Waltham, MA, USA).

\section{Chromatin immuno-precipitation (ChIP)}

ChIP analysis was performed with the ChIP Assay Kit (Millipore-Upstate, Schwalbach, Germany) as described by the manufacturer, isolating genomic DNA fragments generated by sonication, using antibodies anti-NKX2-1 (EP1584Y, Abgent), anti-H2B (53H3, Cell Signaling, Danvers, MA, USA), antiH2Bubl (D11, Cell Signaling), anti-H3K4me3 (mAbcam1012, Abcam, Cambridge, UK) and anti-H3K27me3 (mAbcam6002, Abcam). The subsequent ChIP-PGR analysis was performed using taqpol (Qiagen) oligonucleotides as listed in Table S1 (MWG Eurofins) and thermocycler TGradient (Biometra).

\section{Immunostaining}

To determine intracellular localizations of proteins we performed immunostaining as described previously [23]. We used antibodies anti-NKX2-1 (EP1584Y, Abgent) and anti-SMAD3 (7F3, Abnova, Heidelberg, Germany). Secondary antibodies were purchased from Cell Signaling. For analysis cells were mounted with Vectashield (Vector Laboratories, Burlingame, CA, USA) containing the counterstain DAPI.

\section{Analysis of DNA methylation}

SU-DHL-5 cells were treated with $10 \mu \mathrm{g} / \mathrm{ml}$ trichostatin A (TSA, Sigma) or $10 \mathrm{mM}$ 5-Aza-2'-deoxycytidine (Sigma) for $20 \mathrm{~h}$ before being examined by RQ-PCR. To identify particular methylated cytidines within the CpG island at the HOPX locus, genomic DNA of SU-DHL-5 and SU-DHL-4 (for control) was subjected to bisulfite-conversion and analyzed as described recently [24]. The converted DNA was amplified by PCR using oligonucleotides as listed in Table S1. The PGR products were subcloned into pGEMT-easy (Promega, Madison, WI, USA) and the inserts sequenced (MWG Eurofins). Sequences of 10 clones were displayed and compared using BiQ Analyzer (http://biqanalyzer.bioinf.mpg.de).

\section{Results}

\section{Ectopic expression of NKL homeobox gene NKX2-1 in DLBCL cell line}

Here, we screened by expression profiling 20 leukemia/ lymphoma $\mathrm{T}$ - and B-cell lines for aberrant activities of NKLfamily homeobox genes which are implicated in T-cell leukemia [11]. Our findings confirmed expression of NKL family members in T-ALL cell lines, namely: TLX1 in ALL-SIL, TLX3 in HPBALL, NKX2-5 in PEER and CCRF-CEM, and NKX3-1 in JURKAT, PER-117 and RPMI-8402 (data not shown). In addition, we identified conspicuous expression of NKX2-1 and NKX3-1 in DLBCL cell line SU-DHL-5. Understanding the unexpected activation of NKL genes in a malignant B-cell line was the main focus of this enquiry.

Quantitative expression analysis of NKX2-1 and NKX3-1 by RQ-PCR confirmed their activation in SU-DHL-5 at the RNA level. While NKX3-1 was also expressed in Hodgkin lymphoma (HL), multiple myeloma (MM), B-cell lymphoma (BCL) and additional DLBCL cell lines, NKX2-1 was nearly undetectable in the same set of 25 lymphoma cell lines (Fig. 1A), prompting analysis of NKX2-1 and NKX3-1 in primary cells, including thyroid, lung and particular hematopoietic samples to chart physiological tissue specificity in the blood system. The results confirmed physiological NKX2-1 expression in tissues of the thyroid and the lung, while in all hematopoietic samples analyzed NKX2-1 transcription was undetectable (Fig. 1B). On the other hand, NKX3-1 expression was confirmed in the cell lines SUDHL-5, JURKAT, PER-117 and LNCAP (prostate) and physiologically in tissues of the thyroid and the lung, while the hematopoietic samples of B-cells and BM showed just weak activity (Fig. 1C).

Protein expression of NKX2-1 and NKX3-1 was analyzed by Western blot. While NKX2-1 protein was clearly displayed in SUDHL-5, NKX3-1 protein was not detectable in that cell line (Fig. 1B,C), consistent with post-transcriptional inhibition which was described recently [25]. For this reason we focused our work on regulation and function of homeobox gene NKX2-1, showing ectopic expression at the RNA and protein level in DLBCL cell line SU-DHL-5. To examine the expression of NKX2-1 in primary material we have checked 204 datasets of untreated DLBCL cases deposited in the GEO database of the NCBI belonging to the study of Lenz and coworkers [20]. This screening revealed statistically significant enhanced NKX2-1 activity in 11 (5\%) DLBCL patients (Fig. 1D), supporting the relevance of this oncogenic homeobox gene expression in this malignancy. Of note, the NKX2-1 overexpressing DLBCL patients showed no clear correlation with known disease subsets.

Since deregulated expression of NKL homeobox genes in TALL is primarily caused by chromosomal aberrations, we analyzed the karyotype of SU-DHL-5 by SKY, FISH and in a genomic array with respect to the NKX2-1 gene which is located at $14 \mathrm{q} 13$ (36.9 Mb). However, copy number data (Fig. 1E), gene-specific FISH results (not shown) and SKY results (Fig. 1F) all returned wild type configurations at this locus, discounting a chromosomal mechanism behind the deregulated transcription.

Turning to potential transcriptional regulators which might induce aberrant NKX2-1 activity, we compared expression array data of SU-DHL-5 with 3 control DLBCL cell lines - SU-DHL-4, SU-DHL-10, and SU-DHL-16. After inspection of the top 1000 up- and downregulated genes in SU-DHL-5, potential candidates were shortlisted and functionally categorized as shown in Table 1. This exercise revealed conspicuous involvement of TFs, chromatin and signaling genes which were then subjected to more detailed consideration.

\section{Transcription factors HEY1 and NKX2-1 are mutually exciting}

Among TFs bHLH factor HEYl was particularly intriguing (one of the top 10 upregulated genes). RQ-PCR analysis of a panel of cell lines confirmed high expression levels in SU-DHL-5 (Fig. 2A). However, MM and HL cell lines also showed moderate transcription of HEY1. RQ-PCR analysis of SU-DHL-5 in comparison to primary cells revealed prominent HEY 1 expression in lung, thyroid, and in BM, LN and thymus. These data suggest a functional role of HEY1 in early lymphopoiesis. Peripheral B-cells and PBMCs lacked HEYl transcripts (Fig. 2A), indicating 
A

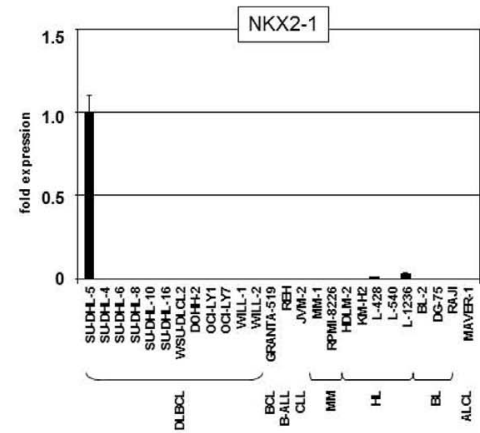

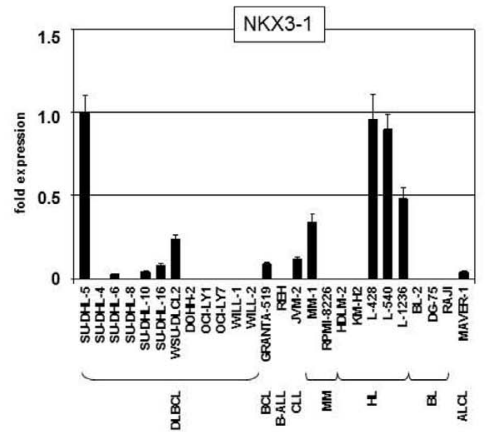

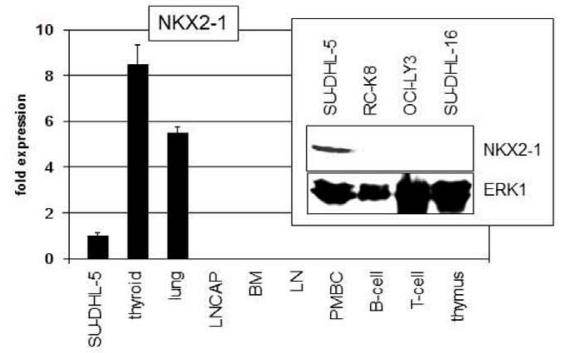

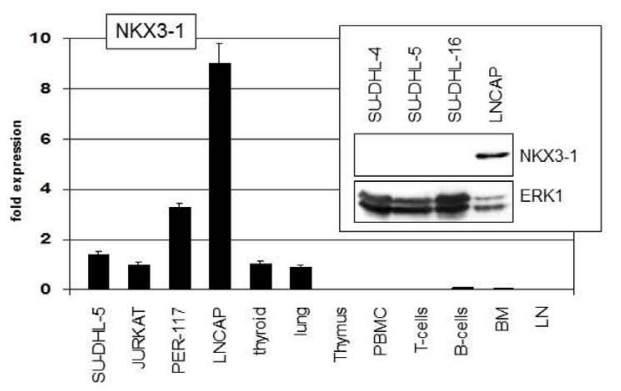

E

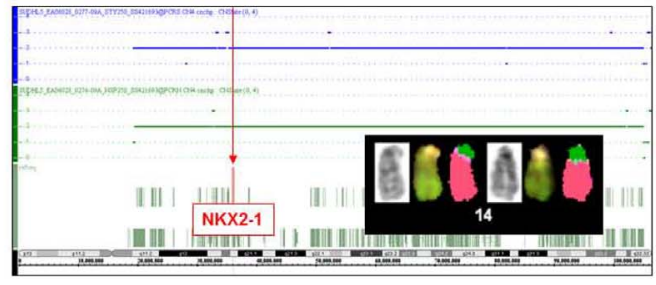

D
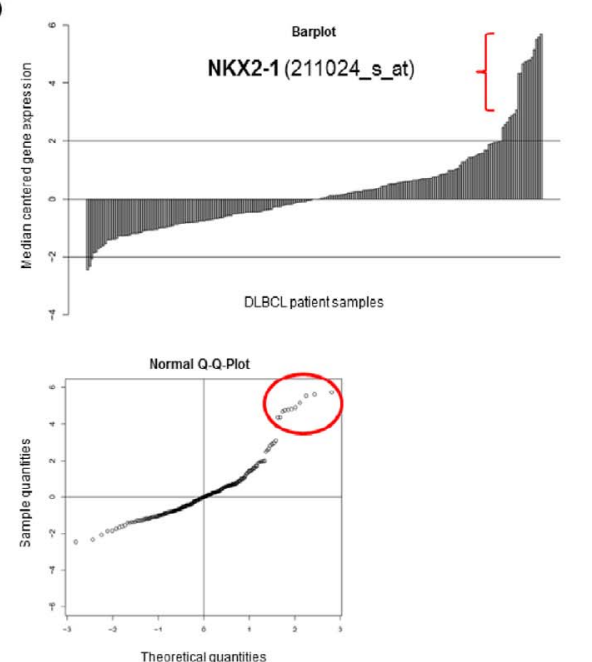

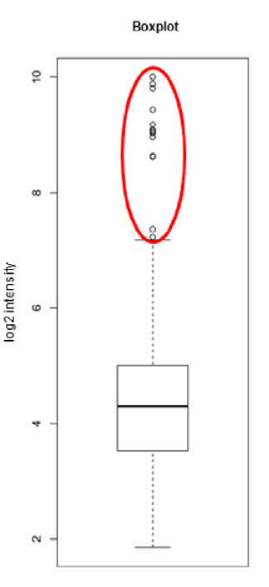

NKX2-1 (211024 s at)

$\mathbf{F}$

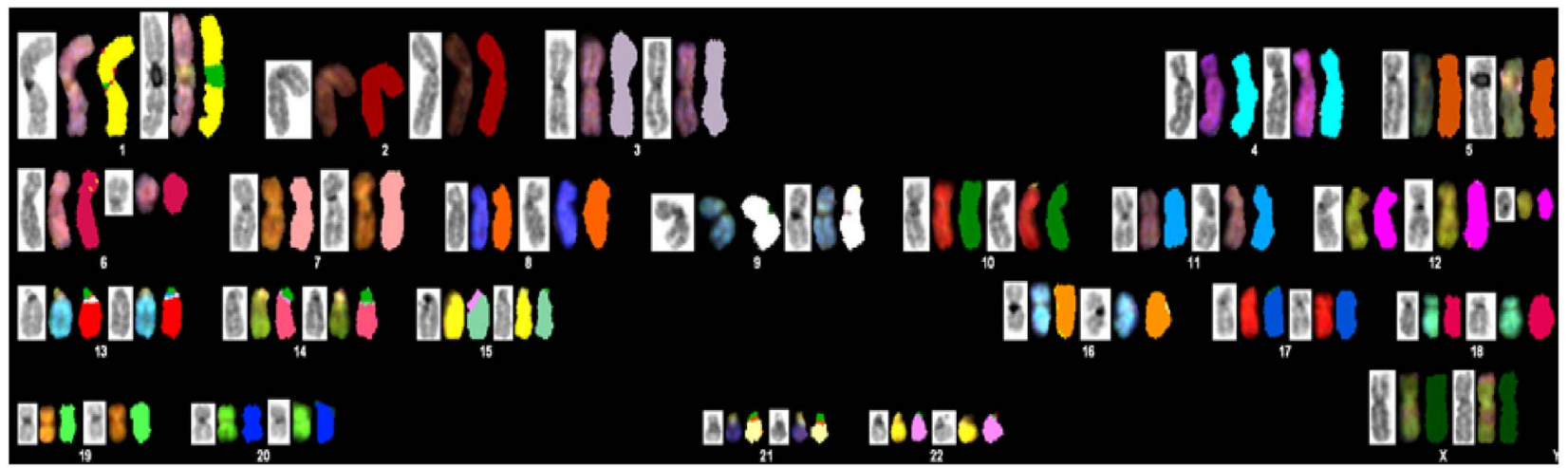

Figure 1. Expression and karyotyping. (A) RQ-PCR analysis of NKX2-1 (left) and NKX3-1 (right) in SU-DHL-5 (expression level was set to unity) and in leukemia/lymphoma control cell lines. (B) RQ-PCR analysis of NKX2-1 in primary cells, demonstrating physiological expression in thyroid and lung and ectopical expression in SU-DHL-5 cells. Western blot analysis (insert) confirms NKX2-1 expression in SU-DHL-5 cells while control DLBCL cell lines RC-K8, OCI-LY3 and SU-DHL-16 are negative. ERK1 was used as loading control. (C) RQ-PCR analysis of NKX3-1 in primary cells and selected cell lines, demonstrating physiological expression in thyroid, lung and prostate and ectopic expression in SU-DHL-5 and T-ALL cell lines JURKAT and PER-117. Western blot analysis (insert) shows NKX3-1 expression in prostate LNCAP cells while SU-DHL-5 lacks detectable NKX3-1 protein. (D) In silico expression analysis of NKX2-1 was performed in 204 DLBCL patient samples (NCBI GEO accession number GSE11318). Median centred gene expressions were illustrated in a barplot (left), showing 11 patients with enhanced expression of NKX2-1 (red bracket). Illustration of these datasets by a boxplot (right) and a Q-Q-plot (below) confirms significantly enhanced expression of NKX2-1 in these 11 patient samples (red circles). (E) Copy number analysis by genomic profiling indicates absence of aberrations at the locus of NKX2-1 at 14q13. The insert shows an enlargement of chromosome 14 obtained by SKY karyotyping. (F) SKY karyotyping of SU-DHL-5 shows absence of rearrangements at chromosome 14 while chromosome 6 demonstrates presence of aberrations. doi:10.1371/journal.pone.0061447.g001 
Table 1. Gene data of SU-DHL-5.

\begin{tabular}{llll}
\hline & & & \\
\hline & Overexpressed & Downregulated & Mutated \\
\hline Transcription & $\begin{array}{l}\text { HEY1, NKX2-1, RUNX1T1, HOXC6, HOXA10, } \\
\text { ETV5, NKX3-1, RUNX2, EGR1, POU2F2, TLE4, } \\
\text { ZHX2 }\end{array}$ & ETS2, TCL6, HLXB9, FLI1, LMO7, BCL11A & \\
Chromatin & HOPX, IGSF4, MLL, AF9, HIST1H3E & $\begin{array}{l}\text { HMGN3, ASB2, JMJD1C, ARID5B, JMJD2B, } \\
\text { PPP1R2, JARID1D, USP46, ARID4A }\end{array}$ & ARID1A, JARID2 \\
Signaling & SMAD9, IL4, STAT3 & SMAD6, IGF1R, BMPR2 & IGF1R, MAP3K14, MAP2K1, MAP3K1, \\
NO/cAMP/cGMP/PDE & DDAH1, PRKAR2B, PRKCE, NOS1 & PDE3B, PDE4A, PDE6D, PDE9A, & AKAP12, PDE4DIP \\
WNT-Signaling & PRKAR2A, KCNQ1, AKAP7 & \\
NFkB-Signaling & SOCDD1, WNT5B, WNT5A, LEF1 & CTNNA1, WNT3, VAV2, SOX4 & \\
Other & DHRS2, EML1, ENO2 & & PTEN \\
\hline
\end{tabular}

Expression profiling data were analyzed and candidates among the top 1000 overexpressed and downregulated genes were selected and sorted according to their functional properties. Furthermore, mutated genes identified by sequence analysis of SU-DHL-5 (provided by the BROAD Institute, www.broadinstitute.org) were selected and sorted as well. The genes are categorized and ordered according their expression level or alphabetically for mutated genes.

doi:10.1371/journal.pone.0061447.t001

downregulation in differentiated B-cells and other leukocytes and thus aberrant upregulation in SU-DHL-5.

To analyze the regulatory impact of HEY1 on NKX2-1 expression we treated SU-DHL-5 cells with siRNA directed against HEY1. Subsequent quantification of HEY1 and NKX2-1 expression demonstrated reduction of both transcripts as compared to cell samples treated with control siRNA (Fig. 2B). Furthermore, overexpression of HEY1 or the related TF HES1 was followed by increased NKX2-1 expression (Fig. 2B). Together, these results show that HEY1 contributes to NKX2-1 expression in DLBCL cell line SU-DHL-5. That HEY1 acts as a transcriptional repressor suggests indirect activation of NKX2-1 probably via inhibition of negative regulators as shown below [26].

Sequence analysis of the promoter region of HEYl identified 3 potential binding sites for NKX2-1 (Fig. 2G), indicating direct regulatory impact of this homeoprotein in HEY1 expression. Subsequent siRNA-mediated knockdown of NKX2-1 inhibited transcription of NKX2-1 and HEY1, confirming regulation by NKX2-1 (Fig. 2C). ChIP analysis using anti-NKX2-1 confirmed direct binding of NKX2-1 to the promoter region of HEY1 (Fig. 2G). These data show that NKX2-1 activates HEY1 transcription directly. Genomic copy number data and SKY analyses excluded genomic aberrations at the HEY1 locus at 8q21 (Fig. S1), highlighting the role of NKX2-1 in HEY1 regulation.

HEY1 and HES1 are prominent targets of the NOTCHpathway in lymphopoiesis [4]. To analyze the potential impact of NOTCH on HEY1 expression we treated SU-DHL-5 cells with the $\gamma$-secretase inhibitor DAPT. Subsequent RQ-PCR analysis showed reduced HEYl levels in treated samples (Fig. 2D), confirming NOTCH regulation. Accordingly, siRNA-mediated knockdown of NOTCH corepressor SPEN enhanced HEYl expression more than twofold (Fig. 2D). Additional findings using DAPT discounted regulation of NKX2-1 by NOTCH-signaling (Fig. 2D).

Expression of Zn-finger homeobox gene 2 (ZHX2) showed elevated levels in SU-DHL-5 as well (Table 1). Comparative RQPGR analysis confirmed high transcript levels in SU-DHL-5, even surpassing primary B-cells (Fig. 2E). Of note, we recently showed reduced expression of ZHX2 in HL cell line L-1236 and an activating input of homeobox gene MSX1 [27]. Accordingly, siRNA-mediated knockdown of MSX1 in SU-DHL-5 reduced transcription of ZHX2 but not of HEY1 (Fig. 2E), contrasting with stimulation of HEY1 by the closely related homeobox gene MSX2 in T-ALL cells [28]. SiRNA-mediated knockdown of homeobox gene NKX2-1 reduced transcript levels of HEY1 as shown above while sparing ZHX2 (Fig. 2E), thus discounting direct regulation of ZHX2 by NKX2-1. However, the precise mechanism of ZHX2 enhancement remains unclear.

\section{Aberrant chromatin structures mediate NKX2-1 expression}

The catalogue of upregulated genes in SU-DHL-5 encoding chromatin components included prominently MLL which is frequently deregulated in leukemia where it activates homeobox genes of the clustered type via H3K4-trimethylation [16]. Quantification of MLL expression in cell lines confirmed elevated RNA levels in SU-DHL-5 in addition to HL cells (Fig. 3A). SiRNA-mediated knockdown of MLL inhibited expression of NKX2-1 but not of HEY1 (Fig. 3B), showing that MLL supports NKX2-1 expression. To analyze corresponding histone modifications at the promoter-regions of NKX2-1 and HEY1 we performed ChIP using antibodies for activatory H3K4me3 (mediated by MLL) and inhibitory H3K27me3 (mediated by EZH2). Analysis of the NKX2-1 promoter showed presence of H3K27me3 in both SU-DHL-4 and SU-DHL-5, while H3K4me3 was restricted to SU-DHL-5 (Fig. 3B). The HEY1 promoter exclusively bore H3K27me3 in both cell lines (Fig. 3B). The presence of both types of analyzed H3-trimethylations at the NKX2-1 locus in SU-DHL-5 indicates aberrant bivalent chromatin configuration known to prime developmental genes for activation and thus likely to favor NKX2-1 expression in this cell line [29]. Moreover, profiling data indicated overexpression of the MLL complex component AF9 and reduced expression of ASB2 which mediates degradation of MLL (Table 1) [17,30].

In leukemia MLL is frequently activated by chromosomal aberrations at 11q23 resulting in amplifications or diverse fusion genes [31]. We looked for chromosomal rearrangements in SUDHL-5 by genomic profiling, finding duplication of MLL accompanied by deletion of its immediately telomeric region (Fig. 3C). FISH analysis confirmed that MLL gain was coupled with the downstream deletion (Fig. 3D). SKY results excluded chromosomal translocations at $11 \mathrm{q} 23$ (Fig. 1D), and RT-PCR analysis of the most prolific MLL-fusion transcripts excluded cryptic fusions with AF4, AF6, AF9, AFX and ENL (Fig. 3E). 
A

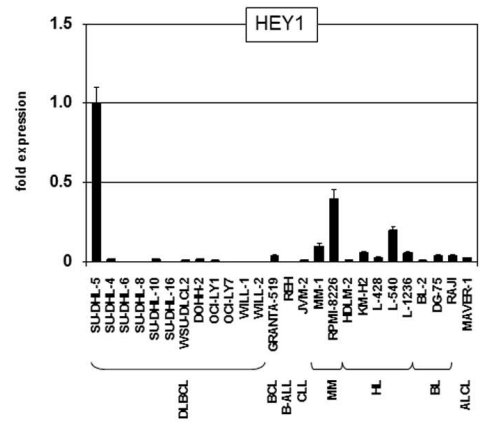

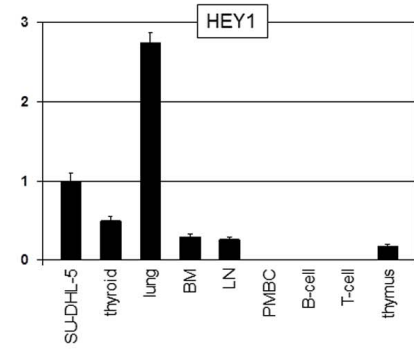
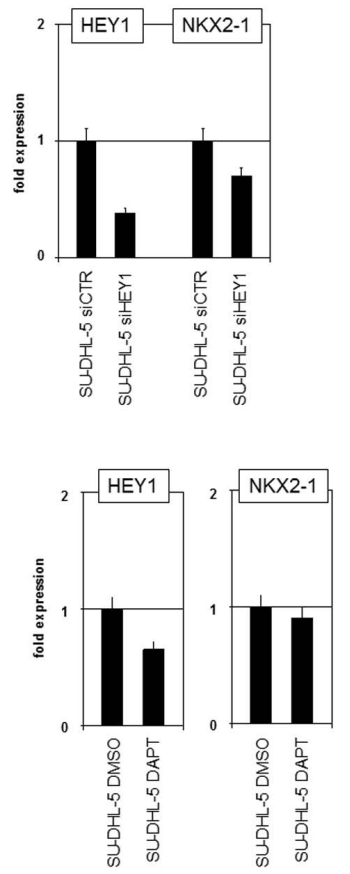
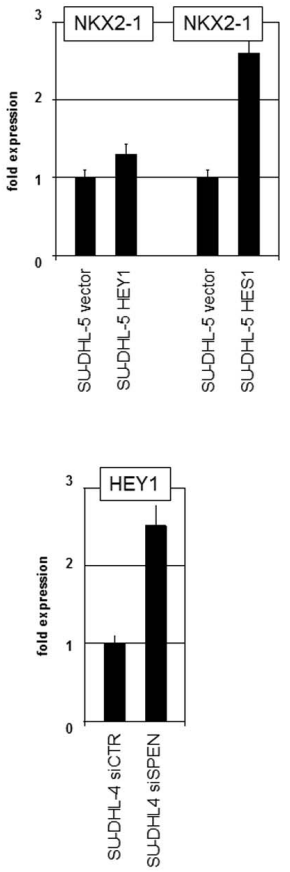

C
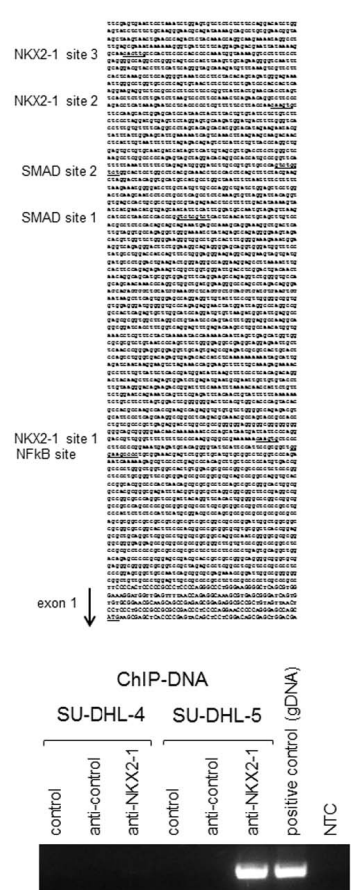

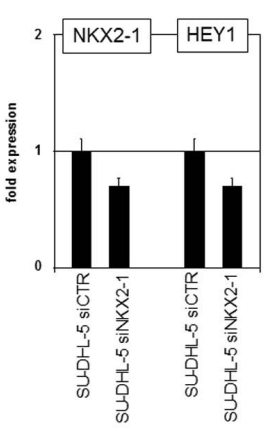

E
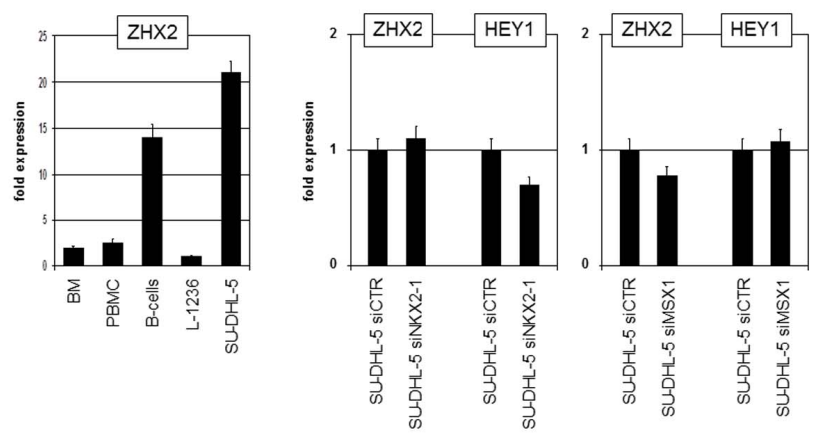

HEY1 promoter
NKK2-1 sites $2+3$

Figure 2. HEY1 and ZHX2. (A) RQ-PCR analysis of HEY1 in SU-DHL-5 (expression level was set to 1) and in leukemia/lymphoma control cell lines (left) and primary cells (right). (B) RQ-PCR analysis of SU-DHL-5 cells treated for siRNA-mediated knockdown of HEY1 (left) and for overexpression of HEY1 and HES1 (right). The results indicate activation of NKX2-1 by HEY1 (and HES1). (C) The promoter sequence of HEY1 contains binding sites for NKX2-1, SMAD and NFkB (left) which are highlighted in bold letters. SiRNA-mediated knockdown of NKX2-1 in SU-DHL-5 cells resulted in reduced expression of HEY1 as shown by RQ-PCR (right, above). ChIP analysis of the HEY1 promoter in SU-DHL-5 and SU-DHL-4 (for control) demonstrated direct binding of NKX2-1 as shown by PCR amplification of genomic fragments (left, below). Untreated genomic DNA served as positive control, NTC: no template control. (D) RQ-PCR analysis of HEY1 and NKX2-1 in SU-DHL-5 cells treated with NOTCH-inhibitor DAPT (left) or with siRNA directed against SPEN (right). (E) RQ-PCR analysis of ZHX2 in cell lines and primary cells (left), and of ZHX2 and HEY1 in siRNA-treated SU-DHL-5 cells (middle and right).

doi:10.1371/journal.pone.0061447.g002

Collectively, our results show that genomic copy number gain of wild type MLL underlies overexpression of MLL in SU-DHL-5 cells.

Furthermore, overexpression of histone H3E (Table 1) correlated with rearrangements of chromosome 6 targeting histone gene cluster 1 (HIST1) at 6p22 as indicated by genomic array data (Fig. 3F). FISH analysis using probes covering HIST1 combined with a painting probe for chromosome 6 confirmed the breakpoint nearby, lying nevertheless outwith the gene cluster (Fig. 3G). The FISH results are consistent with the SKY data showing two derivative chromosomes 6 harboring deletions at $6 \mathrm{p} 22$ and $6 \mathrm{q} 13$, respectively $(\mathbf{F i g}$. 1D, 3F). Quantification of several histone RNA species demonstrated abundant expression of H1C, H2BB, H3E and $\mathrm{H} 3 \mathrm{H}$ in SU-DHL-5 as compared to SU-DHL-4 (Fig. 3H), consistent with coordinate gene activation at the histone locus at 6 p22 by nearby chromosomal rearrangement. Accordingly, SUDHL-5 contained higher levels of core-histone proteins as shown by SDS-PAGE (Fig. 3H). Moreover, histone H2B when analyzed by Western blot showed raised protein expression and enhanced modification with a single ubiquitin at lysine 120, subsequently named H2Bubl (Fig. 3H). The latter is of special interest because H2Bubl reportedly promotes trimethylation of H3K4 by MLL [32], suggesting collaborative activities of the chromosomal aberrations targeting MLL and HIST1.

H2B ubiquitinylation levels are regulated by counteracting ubiquitin-transferases (RNF20, RNF40) and ubiquitin-specific 
A
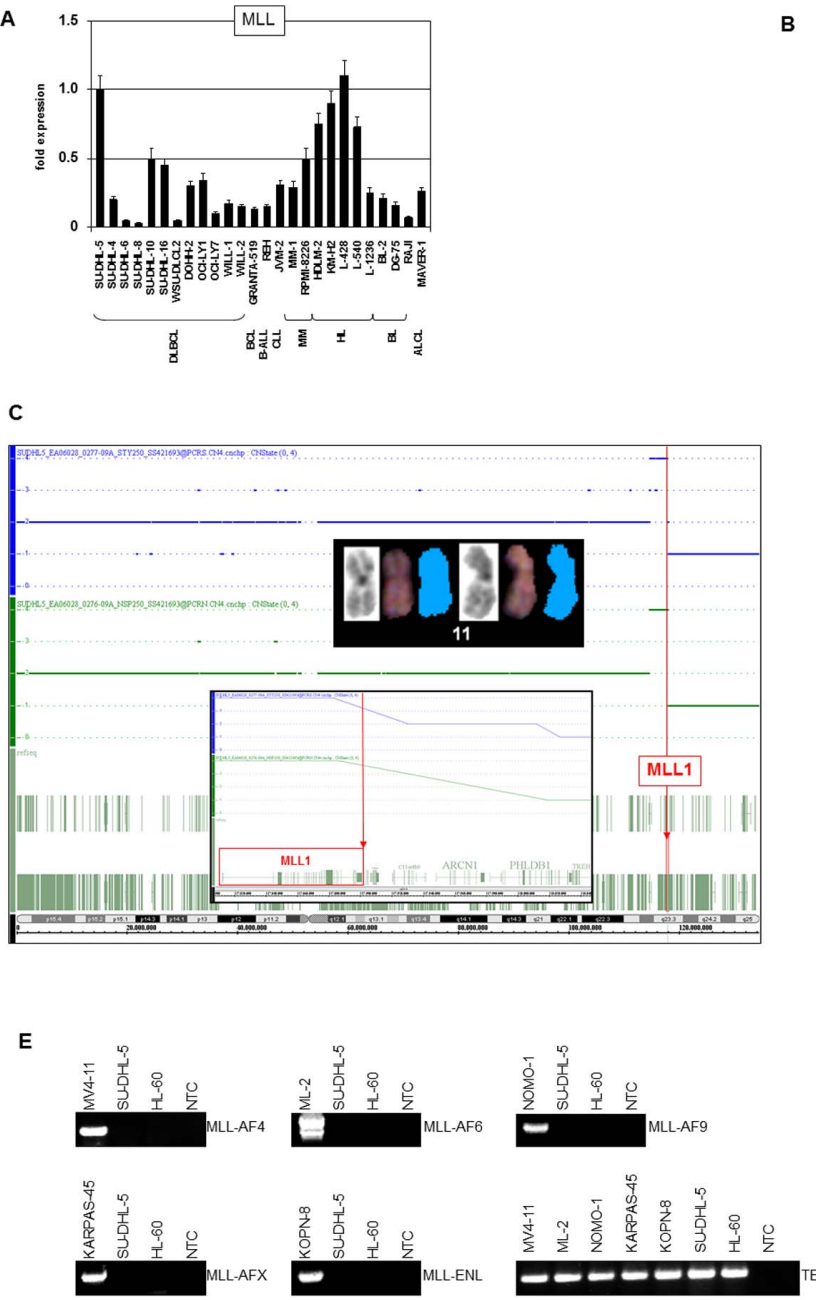

G

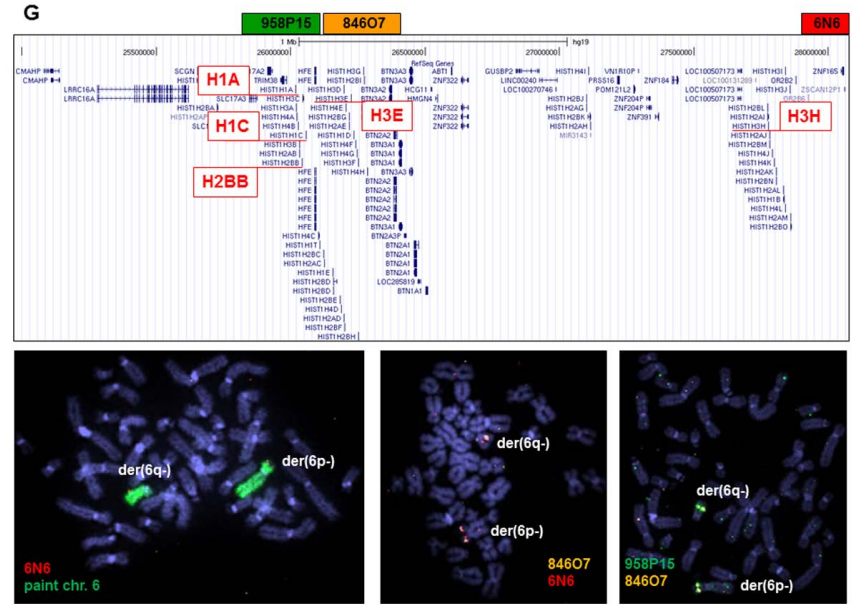

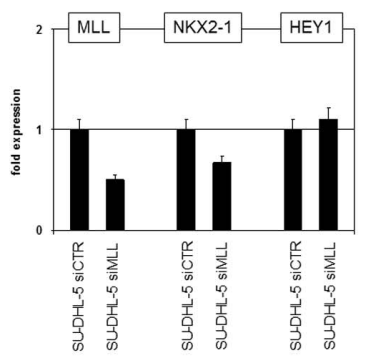

D
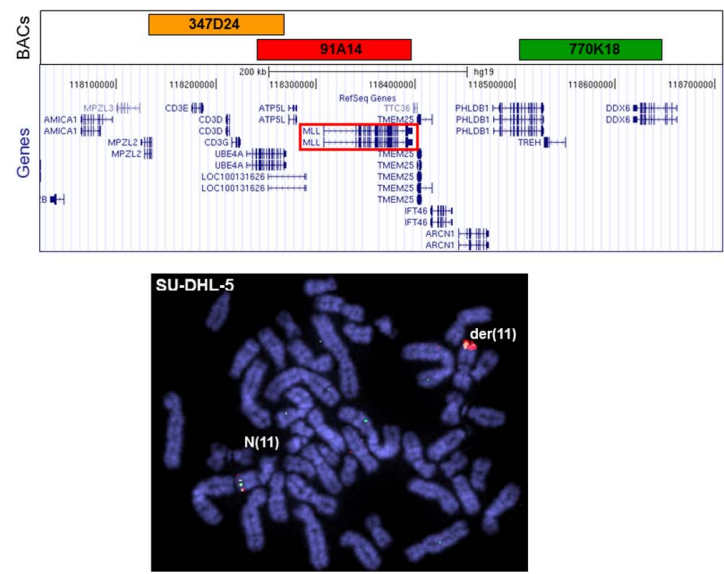

$\mathbf{F}$

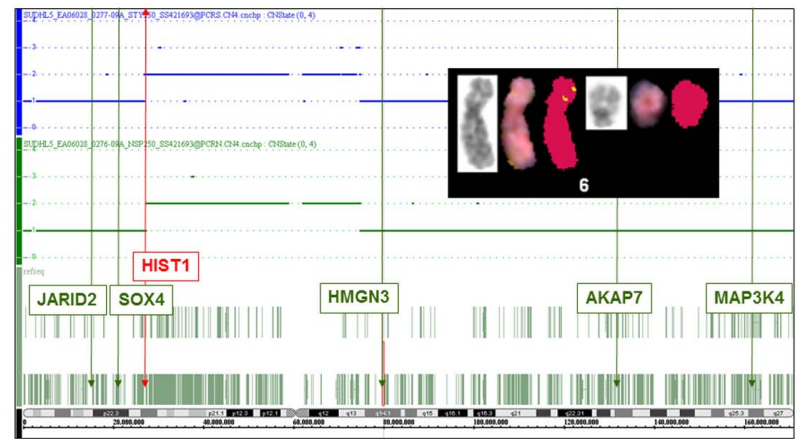

H

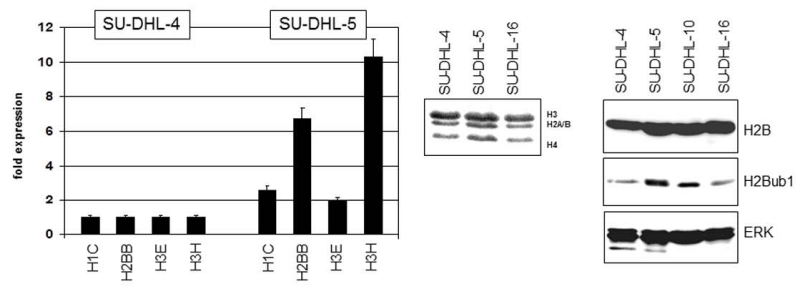

Figure 3. MLL and HIST1. (A) RQ-PCR analysis of MLL in SU-DHL-5 (expression level was set to unity) and in leukemia/lymphoma control cell lines. (B) RQ-PCR analysis of MLL, NKX2-1 and HEY1 in siRNA-treated SU-DHL-5 cells (left). ChIP analysis of the NKX2-1 and HEY1 promoters in SU-DHL-5 and SU-DHL-4 (for control) showed representation of particular histone H3 modifications, as shown by PCR amplification of genomic fragments (right). Untreated genomic DNA served as positive control, NTC: no template control. (C) Copy number analysis by genomic profiling indicates presence of aberrations at MLL at 11q23. Inserts show an enlargement of chromosome 11 obtained by SKY karyotyping and an enlargement of the MLL locus obtained by genomic profiling. (D) FISH analysis of the MLL locus in SU-DHL-5 (below) using BAC probes as indicated above. The results show one wild type allele and one amplified MLL locus. (E) RT-PCR analysis of MLL fusion genes in SU-DHL-5 and particular positive and negative control cell lines. TEL expression served as control, NTC: no template control. (F) Copy number analysis by genomic profiling of chromosome 6 indicates extended deletions at both arms. The HIST1 locus maps to the breakpoint region at 6p22. Genes located in deleted regions include JARID2, SOX4, 
HMGN3, AKAP7 and MAP3K4. Insert shows chromosomes 6 analyzed by SKY karyotyping indicating rearrangements at both chromosomes. (G) FISH analysis of the histone gene cluster HIST1 at 6 p22 (below) using painting probe and BACs as indicated above. (H) RQ-PCR analysis of selected histone genes in SU-DHL-5 and SU-DHL-4 for control (left). PAGE analysis of histone proteins in three DLBCL cell lines (middle) demonstrates elevated levels in SU-DHL-5. Western blot analysis of H2B, H2Bub1 and ERK (for control) in four DLBCL cell lines (right) demonstrates elevated levels in SU-DHL-5. doi:10.1371/journal.pone.0061447.g003

proteases (USPs) [33]. Profiling data indicated repression of USP46 in SU-DHL-5 as confirmed by RQ-PCR analysis in comparison to control cell lines (Fig. 4A). However, USP46 expression was not regulated by the transcriptional repressor HEY1, as analyzed by knockdown and overexpression experiments (Fig. 4A). RQ-PCR analysis of RNF20 and RNF40 showed upregulation in SU-DHL-5 relative to control cell lines $(\mathbf{F i g} . \mathbf{4 B})$. Knockdown of RNF20 and RNF40 by siRNA treatment inhibited expression of NKX2-1 (Fig. 4G) demonstrating that these H2B ubiquitin-transferases support expression of that homeobox gene. Moreover, siRNA-mediated knockdown of NKX2-1 inhibited expression of RNF40 (Fig. 4C), showing that expression of RNF40 is supported by NKX2-1 and thus presence of reciprocal regulation.

These results prompted us to look for additional deregulated histone modifiers which may contribute to the aberrant chromatin structure at NKX2-1. Inhibitory trimethylation of H3K27 is conducted by polycomb repressor complex (PRG) 2 (containing EZH2, JARID2, HOPX, E2F6), and is removed by histone methylase JMJD3 [34,35]. The expression level of JMJD3 was elevated in SU-DHL-5 as shown by RQ-PCR analysis (Fig. 4D). However, JMJD3 was not regulated by NKX2-1 (Fig. 4D). The expression of EZH2 was not significantly altered in SU-DHL-5 as compared to control cell lines (Fig. 4E). Nevertheless, treatment of SU-DHL-5 with EZH2/PRC2 inhibitor DZNep resulted in enhanced transcription of both NKX2-1 and HEY1 (Fig. 4E). These results are consistent with our ChIP data, showing presence of EZH2-mediated H3K27me3 at the promoter regions of both genes (Fig. 3B). Although microarray expression of the gene encoding PRC2 component JARID2 was normal, genomic array data showed monoallelic deletion at 6p22 (Fig. 3F). Sequence data of SU-DHL-5 cells (provided by the BROAD Institute, Table 1) showed mutation of JARID2. Thus SU-DHL-5 is hemizygous for mutated JARID2. Homeobox only protein (HOPX) is associated with PRC2, regulating its capacity for repression $[36,19]$. SU-DHL-5 showed remarkably high levels of HOPX expression as indicated by profiling data (Table 1) and confirmed by RQ-PCR results of lymphoma cell lines $(\mathbf{F i g} . \mathbf{4 F})$. SiRNA-mediated knockdown of HOPX resulted in reduced expression of NKX2-1 (Fig. 4G), showing that HOPX activates NKX2-1 transcription. But this activation was not reciprocal, since reduction of NKX2-1 was unaccompanied by altered HOPX levels (Fig. 4G). However, genomic copy number data and SKY results excluded genomic aberrations at the HOPX locus (Fig. S2). Moreover, examination of DNA methylation of a conspicuous CpG island at the HOPX locus (CpG 109) excluded an abnormal configuration (Fig. S3), leaving the mechanism of this striking overexpression elusive. HOPX is associated with E2F6 which showed reduced expression levels in SU-DHL-5 (Fig. 4H). Interestingly, this reduction was mediated by HEY1 as shown by knockdown experiments which led to overexpression of E2F6 (Fig. 4H). Together, our results demonstrate a significant role for several chromatin modifiers underlying NKX2-1 activation in SUDHL-5. These regulatory interactions are partly reciprocal and constitute feedback-loops, probably resulting in enhanced and stabilized gene activities.

\section{Signaling pathways differentially regulate expression of NKX2-1 and HEY1}

The results of comparative expression analysis in SU-DHL-5 indicated deregulation of several signaling components belonging to a variety of pathways, including $\mathrm{TNFa} / \mathrm{NFkB} / \mathrm{PRKC}, \mathrm{TGFb} /$ BMP/SMAD, IL4/STAT3 and NOS1/cAMP/cGMP/phosphodiesterases (PDEs) which plausibly contribute to NKX2-1 and/or HEYl expression (Table 1). These pathways were analyzed in subsequent experiments.

Treatment of SU-DHL-5 cells with TNFa for $1 \mathrm{~h}$ or $4 \mathrm{~h}$ resulted in activated transcription of both NKX2-1 and HEY1 (Fig. 5A). In accordance with this result, treatment with NFkB inhibitor reduced expression of both genes $(\mathbf{F i g}$. 5B), demonstrating a positive role for $\mathrm{TNFa} / \mathrm{NFkB}$-signaling in transcriptional regulation. Furthermore, expression of protein kinase $\mathrm{C}$ variant $\mathrm{E}$ (PRKCE) was enhanced in SU-DHL-5 when compared to control cell lines (Table 1, Fig. 5G). This may be of interest because the activity of NFkB is regulated by PRKC. SiRNA-mediated reduction of NKX2-1 was accompanied by downregulation of PRKCE (Fig. 5C), indicating a positive regulatory role for this NKL homeobox gene. Sequence analysis of the upstream region of PRKCE (UCSC genome browser, release GRCh37/hg19) revealed a binding site for NKX2-1 at $-25.832 \mathrm{bp}$. ChIP analysis of this site confirmed binding of NKX2-1 antibody, demonstrating direct activation of PRKCE by NKX2-1 (Fig. 5G). This kind of gene regulation represents positive feedback activation which activates involved genes, demonstrating a complex gene regulatory network contributing to ectopic NKX2-1 expression.

Treatment of SU-DHL-5 cells with TGFb resulted in enhanced transcription of both NKX2-1 and HEY1 after 16 h (Fig. 5D). Interestingly, after $1 \mathrm{~h}$ of treatment the expression of HEY 1 raised concentration-dependend while NKX2-1 showed no change in transcript levels at this time point (Fig. 5D). These results may indicate that HEY1 is a direct target of TGFb-signaling, contrasting with the delayed and thus indirect mechanism of NKX2-1 regulation. However, treatment of SU-DHL-5 with inhibitory anti-TGFB showed no effect, excluding autocrine activation (Fig. 5E). SiRNA-mediated knockdown of SMAD3 or SMAD9 reduced transcription of HEY1 while that of NKX2-1 remained unperturbed (Fig. 5F), supporting the direct regulation of HEY1 by TGFb/SMAD-signaling. Of note, according to the UCSC genome browser (release GRCh37/hg19) the promoterregion of HEY 1 contains binding-sites for SMAD proteins which are colocated with those of NKX2-1 (Fig. 2C): a significant observation, since SMAD3 protein has been shown to interact with NKX2-1 [37]. Immunostaining of NKX2-1 and SMAD3 in SU-DHL-5 cells consistently revealed colocalization of both TFs (Fig. 5F). Therefore, our results indicate that in SU-DHL-5 cells NKX2-1 and SMAD3 coactivate HEY1 by protein-protein interaction and direct binding to the promoter region.

The impact of additional pathways in expression of NKX2-1 and HEY1 was analyzed by treatment of SU-DHL-5 cells with IL4, BMP4, IL10 and WNT5B for $16 \mathrm{~h}$ (Fig. 5G). The most significant effects on NKX2-1 were observed after stimulation with IL4 and for HEY1 with BMP4. BMP4 signaling is mediated by SMAD proteins just like $\mathrm{TGFb}$, showing consistent results for HEY1 regulation. IL4-signaling is mediated by STAT3 which is upregulated in SU-DHL-5 (Table 1). Accordingly, siRNA- 
A
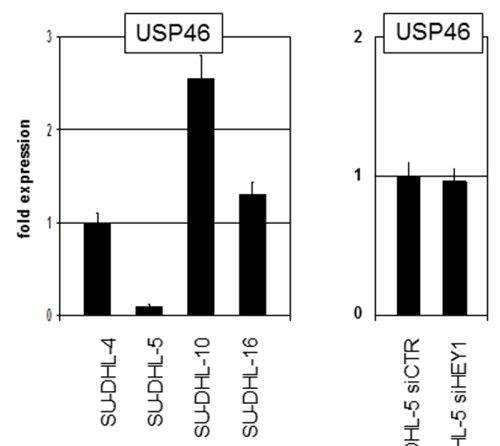

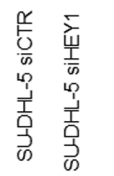

B

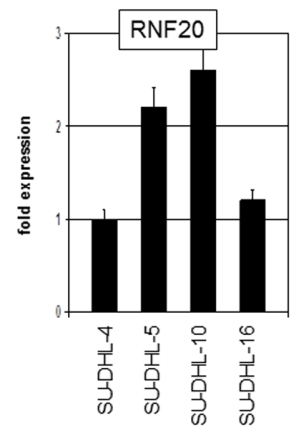

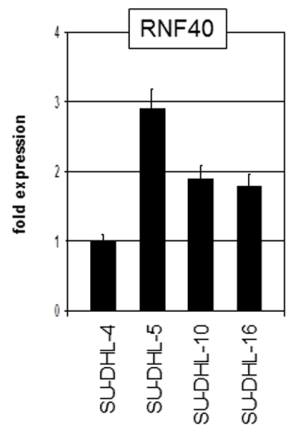

C

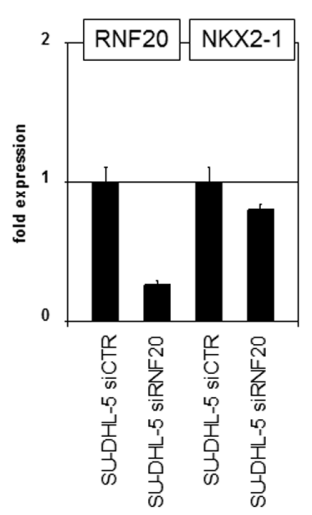

E

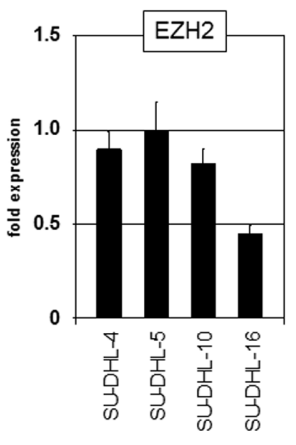

G

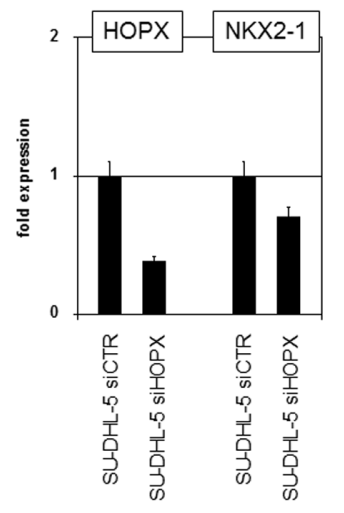

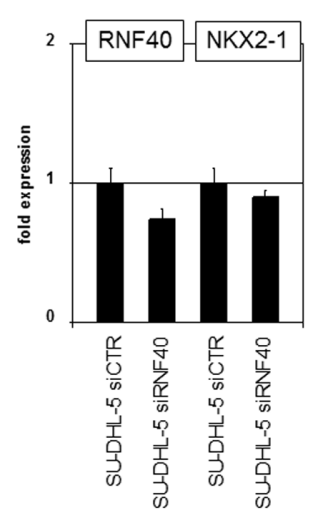

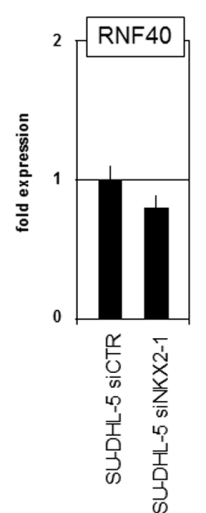

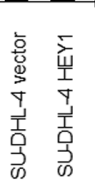

D

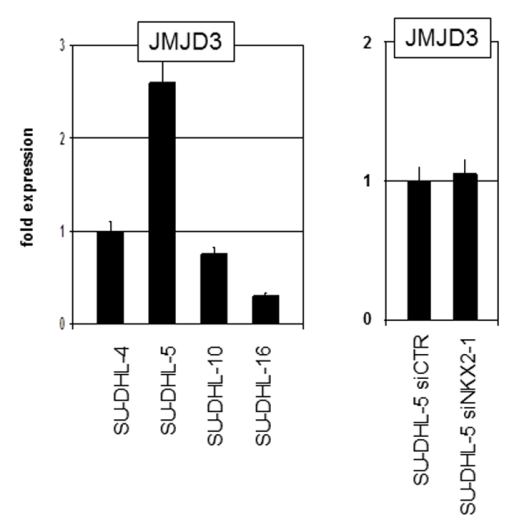

$\mathbf{F}$

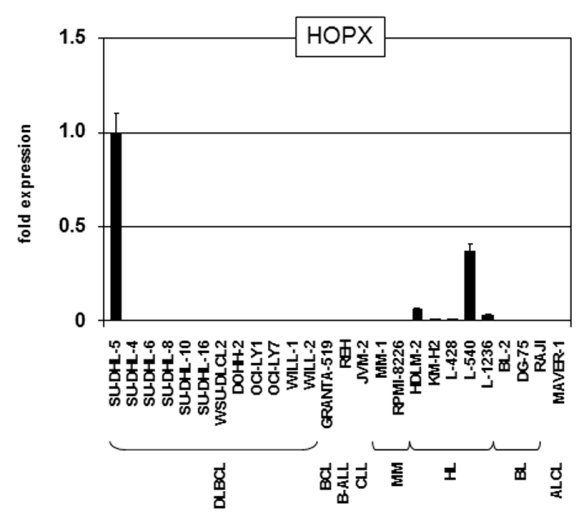

H

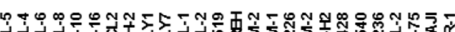

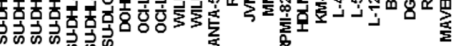

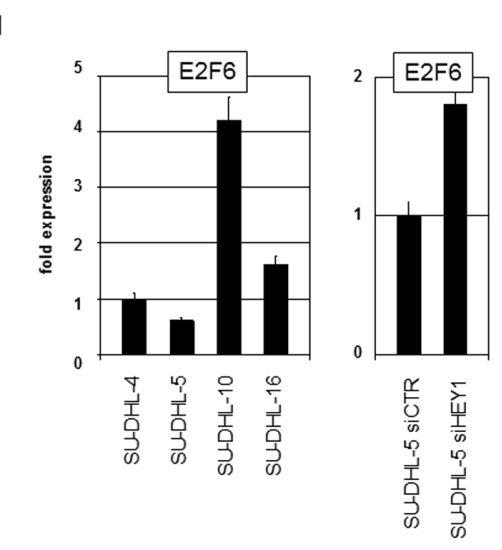

April 2013 | Volume 8 | Issue 4 | e61447
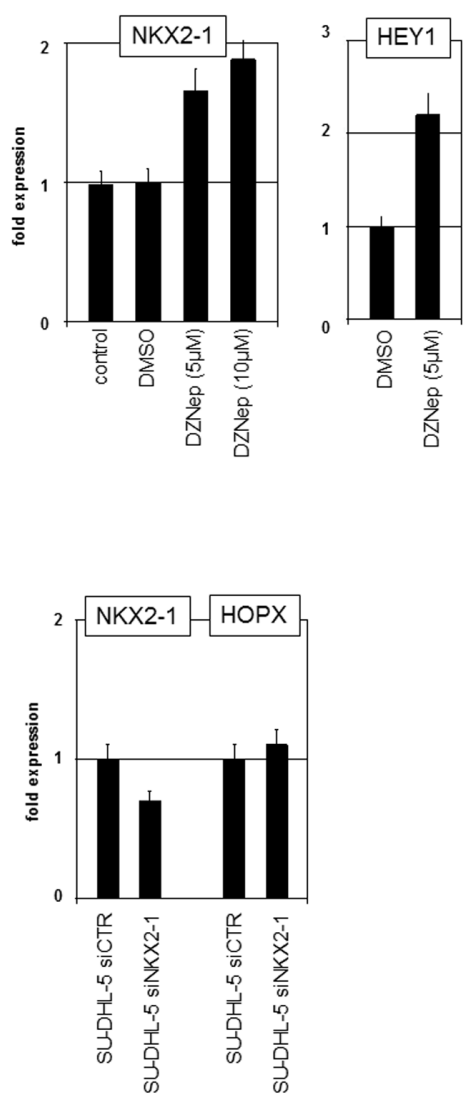
Figure 4. Signaling pathways. (A) RQ-PCR analysis of NKX2-1 and HEY1 in SU-DHL-5 cells treated with TNFa for $1 \mathrm{~h}$ (left) and $4 \mathrm{~h}$ (right). (B) RQPCR analysis of NKX2-1 and HEY1 in SU-DHL-5 cells treated with NFkB-inhibitor for $4 \mathrm{~h}$ (left) and $16 \mathrm{~h}$ (right). (C) RQ-PCR analysis of PRKCE in DLBCL cell lines (left) and in SU-DHL-5 cells treated for siRNA-mediated knockdown of NKX2-1 (middle). ChIP analysis of the PRKCE promoter in SU-DHL-5 and SU-DHL-4 (for control) demonstrated binding of NKX2-1 as shown by PCR amplification of genomic fragments (right, below). Untreated genomic DNA served as positive control, NTC: no template control. (D) RQ-PCR analysis of NKX2-1 and HEY1 in SU-DHL-5 cells treated with TGFb for $1 \mathrm{~h}$ (left) and $16 \mathrm{~h}$ (right). (E) RQ-PCR analysis of NKX2-1 and HEY1 in SU-DHL-5 cells treated with inhibitory antibody directed against TFGb for $16 \mathrm{~h}$ showed no significant differences. (F) RQ-PCR analysis of NKX2-1 and HEY1 in SU-DHL-5 cells treated with siRNA directed against SMAD3 and SMAD9 (above). Immuno-fluorescence analysis of SMAD3 (green) and NKX2-1 (red) in SU-DHL-5 demonstrates nuclear colocalization as compared to the DAPI-stained (blue) nucleus (below). (G) RQ-PCR analysis of NKX2-1 (left) and HEY1 (middle) in SU-DHL-5 cells treated with IL4, BMP4, IL10 and WNT5B for 16 h. RQPCR analysis of NKX2-1 and HEY1 in SU-DHL-5 cells treated for siRNA-mediated knockdown of STAT3 (right). (H) Heatmap of PDE genes in 6 DLBCL cell lines. PDE3B, PDE4A, PDE4B, PDE6D and PDE9A are highlighted. Red indicates high expression levels, green low, and black medium levels. (I) RQPCR analysis of NKX2-1 and HEY1 in SU-DHL-5 cells treated with cAMP, cGMP and sildenafil for $4 \mathrm{~h}$ and $16 \mathrm{~h}$. (K) RQ-PCR analysis of NKX2-1 and HEY1 in SU-DHL-5 cells treated for siRNA-mediated knockdown of PDE6D (left). RQ-PCR analysis of NOS1 in DLBCL cell lines (middle) and in SU-DHL-5 cells treated for siRNA-mediated knockdown of NKX2-1 (right). (L) RQ-PCR analysis of PDE4A and PDE6D in SU-DHL-5 cells treated for siRNA-mediated knockdown of HEY1.

doi:10.1371/journal.pone.0061447.g004

mediated knockdown of STAT3 reduced expression of NKX2-1 while HEY1 transcription was not significantly affected $(\mathbf{F i g} . \mathbf{5 G})$. These results support an activating role for IL4/STAT3-signaling on NKX2-1 and for BMP4/SMAD-signaling on HEY1 in SUDHL-5.

Finally, we analyzed the impact of cAMP/cGMP-signaling in expression of NKX2-1 and HEY1. This pathway stood out due to overexpression of NOS1 (synthesizes nitric oxide activating guanylate cyclase) and reduced expression levels of several PDEs as indicated by comparative profiling data (Table 1) and illustrated by a heatmap for PDE-expression (Fig. 5H). Furthermore, overexpressed DDAH1 (Table 1) encodes an inhibitor of the negative regulator ADMA for NOS1. Therefore, SU-DHL-5 cells were treated with cAMP, cGMP and cGMP-specific PDEinhibitor sildenafil and subsequently quantified for NKX2-1 and HEY1 transcription (Fig. 5I). After $4 \mathrm{~h}$ expression of NKX2-1 rose significantly after treatment with sildenafil, while HEY1 rose with cAMP. After $16 \mathrm{~h}$ expression of NKX2-1 peaked in response to treatment with sildenafil and cGMP, while HEY1 responded maximally to treatment with cAMP and to a lesser extent with cGMP and sildenafil (Fig. 5I). These data suggest that expression of NKX2-1 and HEY1 is primarily regulated via cGMP and cAMP, respectively. Accordingly, siRNA-mediated knockdown of cGMP-specific PDE6D resulted in enhanced expression of NKX21 while HEYl remained unchanged (Fig. 5K). The enhanced expression of NOS1 in SU-DHL-5 as compared to control cell lines was confirmed by RQ-PCR analysis (Fig. 5K). SiRNAmediated knockdown of NKX2-1 resulted in strong reduction of NOS1, indicating an activatory role for NKX2-1 (Fig. 5K). However, ChIP analysis excluded direct binding of NKX2-1 to the promoter region of NOS1 (data not shown), suggesting an indirect activation mechanism. Finally, the transcriptional repressor HEY 1 was found to underly PDE4A repression, while non-participant in PDE6D regulation, as shown by overexpression experiments (Fig. 5L). Thus, deregulated expression of PDEs (PDE6D, PDE4A) and NOS1 via HEY1 and NKX2-1 indicate feedback regulation of both TFs.

\section{Discussion}

In DLBCL cell line SU-DHL-5 we have identified ectopic expression of NKX2-1 which is activated by bHLH TF HEY1, aberrant modifications of the chromatin structure, and particular signaling pathways. NKX2-1 belongs to the NKL family of homeobox genes which is implicated in the tumorigenesis of $\mathrm{T}$ ALL $[1,38]$. In silico expression analysis of patient samples indicated aberrant activity of NKX2-1 in 5\% of DLBCL, representing a hitherto unrecognized subgroup of this disease.
Therefore, our results expand the oncogenic role of this gene family within the entity of lymphoid malignancies.

NKX2-1 is physiologically expressed in the developing lung and thyroid but not, as shown here, in hematopoietic cells. In a physiological context NKX2-1 regulates differentiation processes both during embryogenesis and in the adult $[7,8]$. In lung cancer NKX2-1 performs the role of a lineage-specific oncogene enhancing proliferation and survival $[39,40]$. Overexpression of NKX2-1 mediated by genomic amplification enhances tumorigenicity of lung cancer cells as evidenced by colony formation of lung epithelial cells and advanced malignancy in affected patients [41]. Furthermore, NKX2-1 enhances together with FOXA1 survival in lung adenocarcinoma by transcriptional activation of LMO3 [42]. However, we have neither experimentally assayed tumorigenicity, nor survival of SU-DHL-5 cells, but our comparative expression data gave no hint for deregulation of proliferation or apoptosis. Accordingly, SU-DHL-5 showed no increased expression of LMO3, suggesting absence of this particular survival-pathway. Rather, the profiling data of SU-DHL-5 identified TFs and signaling pathways highlighting the view of deregulated cell differentiation mediated by NKX2-1.

Our data ruled out aberrant activation of NKX2-1 via chromosomal rearrangements contrasting with the picture of NKL homeo-oncogenes in T-ALL. We identified several (deregulated) genes involved in NKX2-1 expression by comparative profiling and subsequent knockdown and overexpression studies. Among TFs we identified activating HEYl which underlies NKX2-1 transcription. HEY1 belongs to the inhibitory subgroup of bHLH proteins, deregulation of which promotes the development of leukemia/lymphoma affecting the function of E2A in driving lymphoid development [26,36,43-45]. Our data show direct activation of HEY1 by NKX2-1 and indirect activation of NKX2-1 by repressive HEY1. HEY1 is physiologically expressed in developing lung tissue like NKX2-1 $[7,46]$. Therefore, this regulatory role may also figure in the physiological context of the lung. However, forced expression of HEY1 in DLBCL cell lines did not induce NKX2-1 transcription, indicating that additional factors or chromatin modifications are necessary for the gene activity as described below.

MLL contributes to enhanced expression of NKX2-1 in SUDHL-5 cells. It is overexpressed in this cell line via chromosomal rearrangements resulting in duplication of the wild type gene. Tandem triplication of MLL has been described in intravascular large B-cell lymphoma suggesting a more widespread oncogenic role in B-cell lymphomas than hitherto supposed [47]. The MLL gene encodes a methyltransferase which modifies histone H3 (H3K4me3). This modification marks active chromatin and gene transcription [17]. The presence of both activatory H3K4me3 and 
A

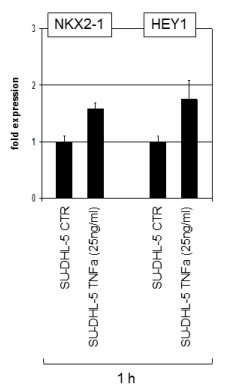

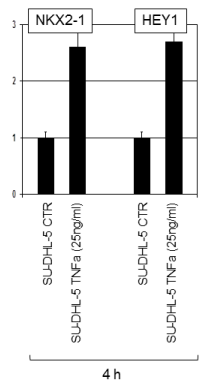
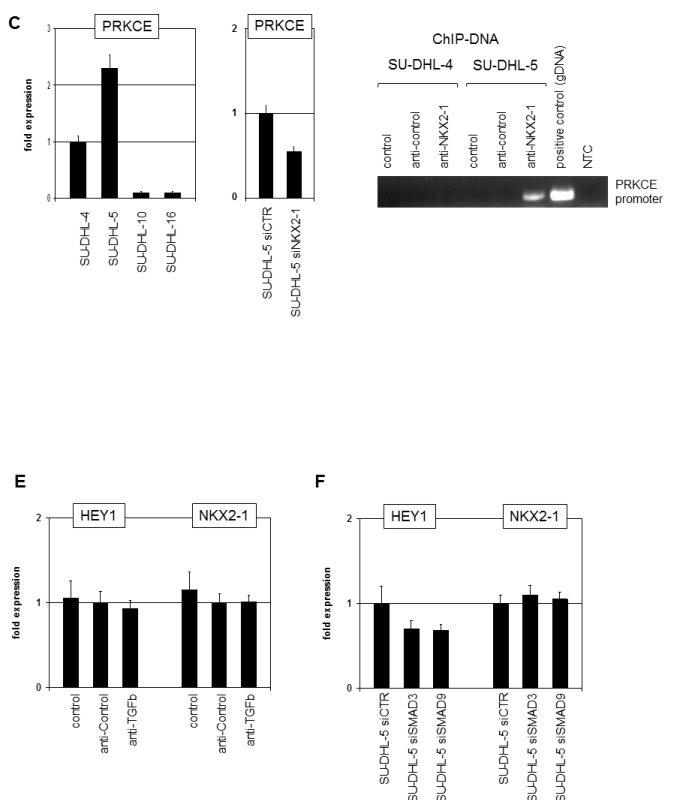
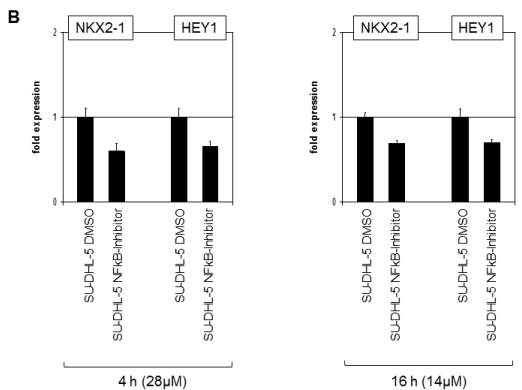

D
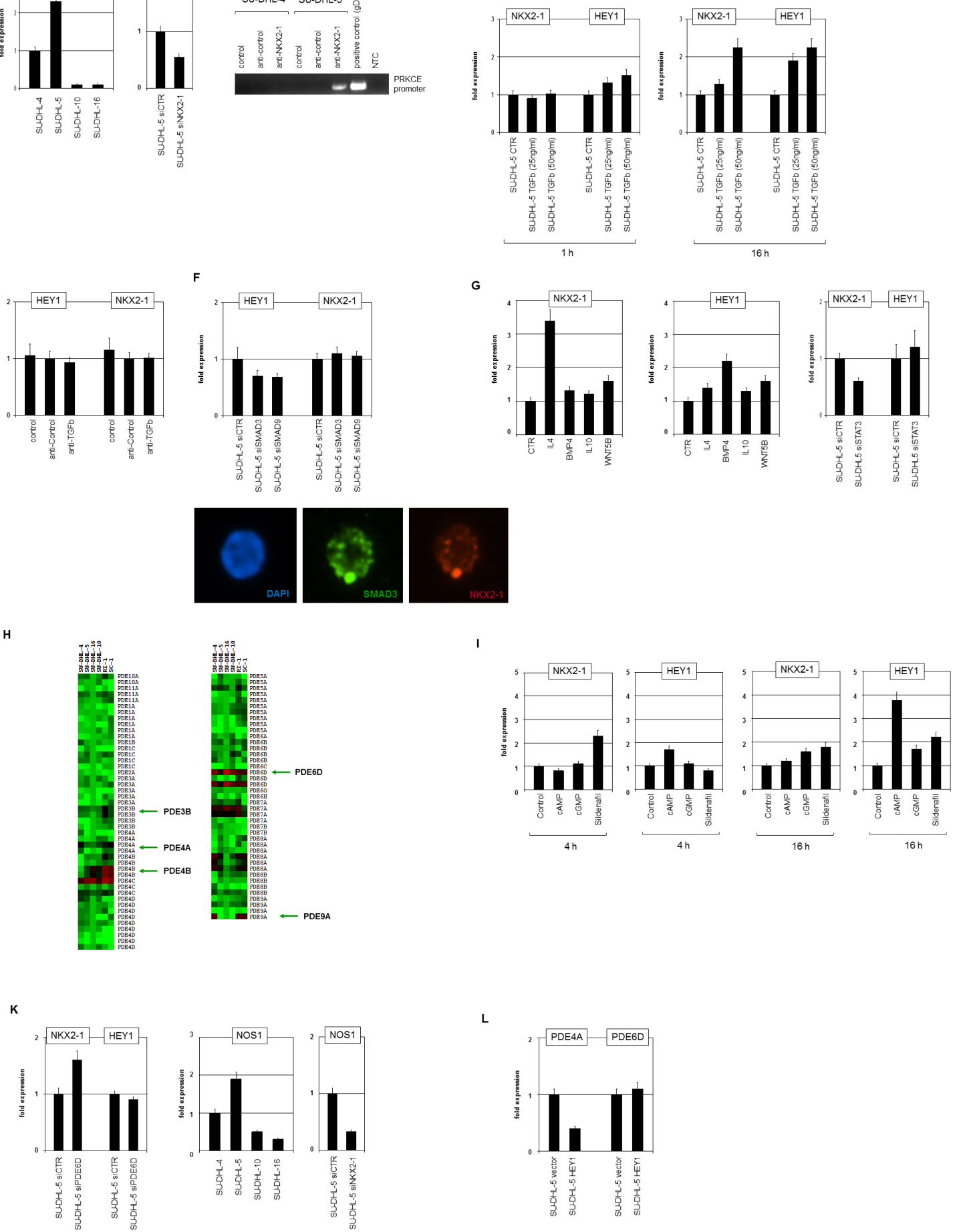
Figure 5. Signaling pathways. (A) RQ-PCR analysis of NKX2-1 and HEY1 in SU-DHL-5 cells treated with TNFa for $1 \mathrm{~h}$ (left) and $4 \mathrm{~h}$ (right). (B) RQPCR analysis of NKX2-1 and HEY1 in SU-DHL-5 cells treated with NFkB-inhibitor for $4 \mathrm{~h}$ (left) and $16 \mathrm{~h}$ (right). (C) RQ-PCR analysis of PRKCE in DLBCL cell lines (left) and in SU-DHL-5 cells treated for siRNA-mediated knockdown of NKX2-1 (middle). ChIP analysis of the PRKCE promoter in SU-DHL-5 and SU-DHL-4 (for control) demonstrated binding of NKX2-1 as shown by PCR amplification of genomic fragments (right, below). Untreated genomic DNA served as positive control, NTC: no template control. (D) RQ-PCR analysis of NKX2-1 and HEY1 in SU-DHL-5 cells treated with TGFb for $1 \mathrm{~h}$ (left) and $16 \mathrm{~h}$ (right). (E) RQ-PCR analysis of NKX2-1 and HEY1 in SU-DHL-5 cells treated with inhibitory antibody directed against TFGb for $16 \mathrm{~h}$ showed no significant differences. (F) RQ-PCR analysis of NKX2-1 and HEY1 in SU-DHL-5 cells treated with siRNA directed against SMAD3 and SMAD9 (above). Immuno-fluorescence analysis of SMAD3 (green) and NKX2-1 (red) in SU-DHL-5 demonstrates nuclear colocalization as compared to the DAPI-stained (blue) nucleus (below). (G) RQ-PCR analysis of NKX2-1 (left) and HEY1 (middle) in SU-DHL-5 cells treated with IL4, BMP4, IL10 and WNT5B for 16 h. RQPCR analysis of NKX2-1 and HEY1 in SU-DHL-5 cells treated for siRNA-mediated knockdown of STAT3 (right). (H) Heatmap of PDE genes in 6 DLBCL cell lines. PDE3B, PDE4A, PDE4B, PDE6D and PDE9A are highlighted. Red indicates high expression levels, green low, and black medium levels. (I) RQPCR analysis of NKX2-1 and HEY1 in SU-DHL-5 cells treated with CAMP, cGMP and sildenafil for $4 \mathrm{~h}$ and $16 \mathrm{~h}$. (K) RQ-PCR analysis of NKX2-1 and HEY1 in SU-DHL-5 cells treated for siRNA-mediated knockdown of PDE6D (left). RQ-PCR analysis of NOS1 in DLBCL cell lines (middle) and in SU-DHL-5 cells treated for siRNA-mediated knockdown of NKX2-1 (right). (L) RQ-PCR analysis of PDE4A and PDE6D in SU-DHL-5 cells treated for siRNA-mediated knockdown of HEY1.

doi:10.1371/journal.pone.0061447.g005

inhibitory H3K27me3 as detected here in SU-DHL-5 at NKX2-1 has been termed "bivalent chromatin modification"-a structure which primes developmental genes for activation in embryonal stem cells [29]. Therefore, this histone-mark may represent one of the basic factors predisposing to ectopic NKX2-1 activation.

Additionally, overexpression of core-histones in SU-DHL-5 coincided with a chromosomal aberration at 6 p22 housing the histone gene cluster 1 . H2B in particular was shown to be overexpressed and strongly ubiquitinated at position K120. This modification guides and enhances the process of MLL-mediated H3K4-methylation, indicating cooperation of both types of chromosomal rearrangements in NKX2-1 expression in SUDHL-5 [32].

Several enzymes performing histone-modifications were deregulated in SU-DHL-5, contributing to a permissive chromatin structure at the NKX2-1 gene. RNF and USP genes encode ubiquitin-specific transferases and proteases, respectively, regulating ubiquitination of histone H2B [33]. Our results demonstrate, in addition to aberrant expression, their impact on deregulating NKX2-1 transcription. Accordingly, NKX2-1 has been described as a target gene of RNF20 in HELA cells [48]. PRC2 contains H3methyltransferase EZH2 and the modulating components HOPX and E2F6 [49,50]. Expression levels of HOPX and E2F6 were altered in SU-DHL-5 and functional analyses demonstrated their impact in NKX2-1 regulation. However, while HOPX expression is activated by NKX2-1 in lung cancer cells, it was not regulated by NKX2-1 in SU-DHL-5 cells [51]. Of note, both HOPX and E2F6 are overexpressed in HL indicating the presence therein of deregulated chromatin structures, albeit distinct from those in SUDHL-5 [52,23]. Interestingly, in SU-DHL-5 many genes encoding deregulated histone modifiers are influenced by NKX2-1 or HEY1 in their expression levels, revealing a reciprocal network which mutually reinforces aberrant oncogene activities.

As well as TFs and chromatin-modifiers we identified signaling pathways regulating NKX2-1 expression: firstly, TNFa, NFkB and the NFkB-activating kinase PRKCE were involved in activation of both NKX2-1 and HEY1; second, IL4/STAT3-signaling which was primarily engaged in the activation of NKX2-1; and finally, $\mathrm{TGFb} / \mathrm{BMP} 4$ and SMAD3 which activated transcription of just HEY1. This last named activity may explain both the presence of

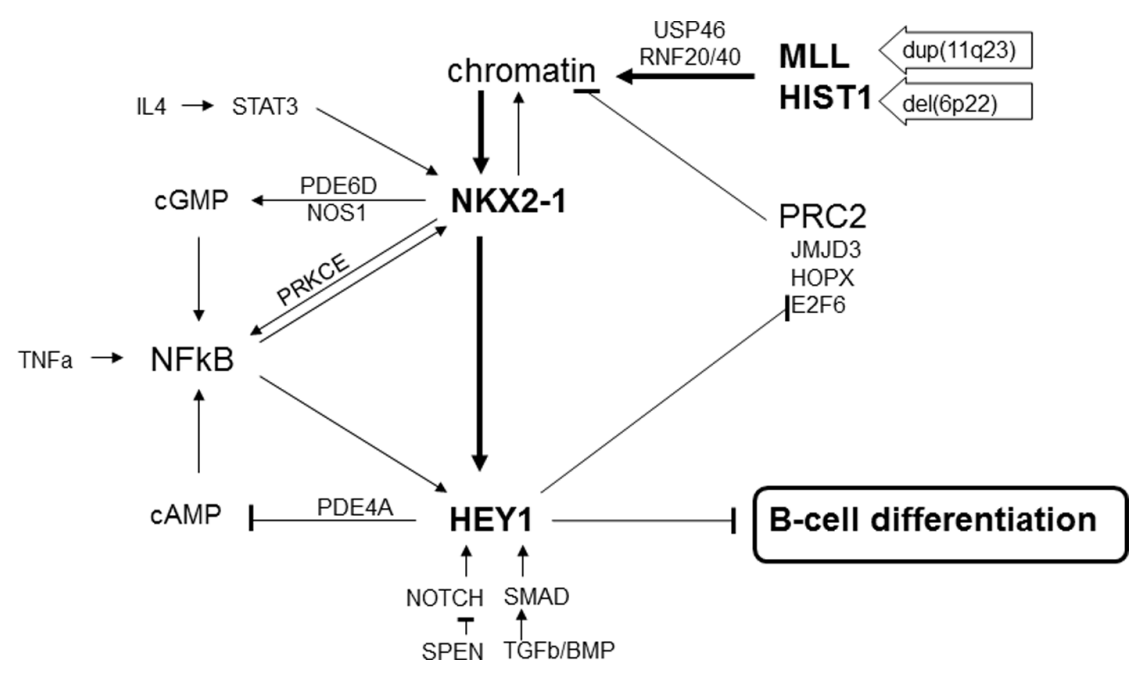

Figure 6. Oncogenic network of NKX2-1. The figure summarizes the regulations of the genes described in this study, highlighting a central position of NKX2-1. Chromosomal aberrations activate expression of MLL (11q23) and histones including H2B (6p22). MLL together with H2Bub1 generate an activatory chromatin structure at NKX2-1. This structure is reinforced by reduced expression of USP46 and E2F6 and elevated expression of RNF20/40, JMJD3 and HOPX, and mediates activation of NKX2-1. NKX2-1 in turn activates directly expression of HEY1 which performs inhibition of B-cell differentiation. Both, NKX2-1 and HEY1 contribute to the activatory chromatin structure by regulating RNF40 and E2F6, respectively. IL4/STAT3signaling enhances expression of NKX2-1. TNFa, CGMP, CAMP and PRKCE support NFkB which activates both NKX2-1 and HEY1. Reduced expression of PDE6D and enhanced expression of NOS1 contribute to elevated CGMP. NOS1 and PRKCE are activated by NKX2-1. HEY1 mediates reduced expression of PDE4A resulting in elevated cAMP levels. Finally, NOTCH-signaling and TGFb-signaling (via SMAD) activate HEY1 expression. SMAD and NKX2-1 interact and coactivate HEY1 transcription.

doi:10.1371/journal.pone.0061447.g006 
neighboring binding sites for NKX2-1 and SMAD seen at the HEY1-promoter, and nuclear colocalization of NKX2-1 and SMAD3 in SU-DHL-5, indicating cooperative activation of HEY1. This coactivation may represent a switch-like regulation which stabilizes gene activities [53]. Furthermore, enzymes regulating levels of cGMP and cAMP were identified as respective mediators of NKX2-1 and HEY1 expression, including NOS1 and specific PDEs. Mutated genes of this pathway in SU-DHL-5 include PDE4DIP and AKAP12. Of note, while reduced levels of PDEs were identified here in a DLBCL cell line, enhanced levels of PDE5A have been reported in HL cells [54], suggesting that PDE-activity may be critical for lymphomagenesis. Furthermore, sequence data revealed several mutated MAP kinases, e.g. MAP3K14, MAP2K1, and MAPK4. However, their impact in NKX2-1 expression was not considered in this study.

Our data plot the emergence of an aberrant gene regulatory network with NKL homeobox gene NKX2-1 occupying a central role (Fig. 6). It comprises several network modules with feed-back motifs. The functional data indicate that these modules contribute to an enhancement and stabilization of NKX2-1 expression. In SU-DHL-5 deregulated chromatin may represent the initial step in NKX2-1 activation and subsequent cell transformation. According to such a model, chromosomal aberrations enhancing MLL and histones poised chromatin at the NKX2-1 locus for activation which subsequently regulates HEY1. This combination of modified chromatin and ectopic expression of transcriptional regulators represents an alternative mechanism of aberrant NKL homeobox gene activation in lymphoid malignancy. In T-ALL deregulation of select NKL family genes is typically effected by chromosomal alterations which juxtapose enhancer elements cognate to T-cell receptor genes or BCL11B [12]. In the case of NKX3-1, however, activation in T-ALL is controlled by particular deregulated hematopoietic TFs (TAL1, LYL1, MSX2) where aberrant chromatin structures may also participate $[13,14]$.

Transdifferentiation or reprogramming of cells is practicable in several cell types including hematopoietic cells and may be effected by forced expression of particular TFs [55]. For example CEBPA and GATAl drive the differentiation into macrophages and megakaryocytes, respectively [56,57], and NKX2-1 together with PAX8 mediate differentiation of embryonic stem cells into thyroid cells [58]. However, aberrant or ectopic expression of oncogenic (cell-type specific) TFs does not result in transdifferentiation of the tumor cells. These TFs rather disturb the physiological process of terminal differentiation, resulting in developmental arrest at immature stages. Explanations for the preference for differentiation arrest instead of reprogramming may be the cellular context as described for the TF TAL1 in T-ALL which resides at different binding sites in normal and leukemic cells or the need of stagespecific coregulators in addition to master factors [59,60]. It is also likely that transdifferentiation requires permissive chromatin states normally present in embryonic cells which may be partially recapitulated in adult cells by treatment with histone methyltransferase inhibitors [61]. Noteworthy in this context is that the expression level of NKX2-1 was about 8-fold higher in primary

\section{References}

1. Chan WJ (2010) Pathogenesis of diffuse large B cell lymphoma. Int J Hematol. 92(2): 219-230.

2. Alizadeh AA, Eisen MB, Davis RE, Ma C, Lossos IS, et al. (2000) Distinct types of diffuse large B-cell lymphoma identified by gene expression profiling. Nature. 403(6769): 503-511.

3. Aifantis I, Raetz E, Buonamici S (2008) Molecular pathogenesis of T-cell leukaemia and lymphoma. Nat Rev Immunol. 8(5): 380-390.

4. Aster JC, Blacklow SC, Pear WS (2011) Notch signalling in T-cell lymphoblastic leukaemia/lymphoma and other haematological malignancies. J Pathol. 223(2): $262-273$. physiological tissues as compared to SU-DHL-5. This scale of difference has been recognized for deregulated NKX2-5 and NKX3-1 in T-ALL as well [14], suggesting oncogenic actions of ectopic NKL homeobox genes at low expression levels instead of driving differentiation at higher levels. This interrelation suggests that enhancement of ectopic oncogene expression (e.g. NKX2-1 in DLBCL) may result in transdifferentiation of the lymphoma cells into benign non-hematopoietic cells, representing a novel concept for cancer therapy.

Taken together, we have identified aberrant expression of NKL homeobox gene NKX2-1 in subsets of DLBCL which is mediated by particular factors including TFs, chromatin mediators, and signaling components. This result expands the oncogenic role of this homeobox gene family within the group of lymphoid malignancies. However, diagnostic and/or therapeutic potentials require additional examinations. Nevertheless, our data may also be of interest for analyses and assessment of NKX2-1 in lung and thyroid cancer.

\section{Supporting Information}

Figure S1 Copy number and SKY data of HEY1. Copy number analysis by genomic profiling indicates absence of aberrations at the locus of HEY1 at 8q13 in SU-DHL-5. The insert shows an enlargement of chromosome 8 obtained by SKY karyotyping.

(TIF)

Figure S2 Gopy number and SKY data of HOPX. Copy number analysis by genomic profiling indicates absence of aberrations at the locus of HOPX at $4 \mathrm{q} 12$ in SU-DHL-5. The insert shows an enlargement of chromosome 4 obtained by SKY karyotyping.

(TIF)

Figure S3 Methylation data for HOPX. The locus of HOPX contains a CpG-island (CpG 109) as shown at the UCSC genome browser (above). RQ-PCR analysis of SU-DHL-5 cells treated with histone deacetylase-inhibitor TSA and DNAmethyltransferase-inhibitor 5-Aza-2'-deoxycytidine (AZA) (right). Data obtained by sequence analysis of bisulfite-treated DNA of SU-DHL-5 and SU-DHL-4 show no significant difference, demonstrating absence of HOPX deregulation via demethylated DNA at CpG 109. Each lollipop represents a CpG; filled lollipops represent methylated CpGs.

(TIF)

Table S1 Oligonucleotides used for PCR. (DOC)

\section{Author Contributions}

Conceived and designed the experiments: SN. Performed the experiments: CM MK RAFM. Analyzed the data: SE JT HQ. Contributed reagents/ materials/analysis tools: HGD. Wrote the paper: SN.

5. Holland PW, Booth HA, Bruford EA (2007) Classification and nomenclature of all human homeobox genes. BMC Biol. 2007;5:47.

6. Garcia-Fernàndez J (2005) The genesis and evolution of homeobox gene clusters. Nat Rev Genet 6(12): 881-892.

7. Boggaram V (2009) Thyroid transcription factor-1 (TTF-1/Nkx2.1/TITF1) gene regulation in the lung. Clin Sci (Lond). 116(1): 27-35.

8. Fagman H, Nilsson M (2011) Morphogenetics of early thyroid development. J Mol Endocrinol. 46(1): R33-42.

9. Ponticos M (2010) The role of the homeodomain transcription factor Nkx2-5 in the cardiovascular system. In: Adv.Vasc.Med., Abraham et al. (eds.) 
10. Shen MM, Abate-Shen C (2003) Roles of the Nkx3.1 homeobox gene in prostate organogenesis and carcinogenesis. Dev Dyn. 228(4): 767-778.

11. Homminga I, Pieters R, Meijerink JP (2012) NKL homeobox genes in leukemia. Leukemia. 26(4): 572-581.

12. Graux C, Cools J, Michaux L, Vandenberghe P, Hagemeijer A (2006) Cytogenetics and molecular genetics of T-cell acute lymphoblastic leukemia: from thymocyte to lymphoblast. Leukemia. 20(9): 1496-1510.

13. Kusy S, Gerby B, Goardon N, Gault N, Ferri F, et al. (2010) NKX3.1 is a direct TAL1 target gene that mediates proliferation of TAL1-expressing human T cell acute lymphoblastic leukemia. J Exp Med. 207(10): 2141-2156.

14. Nagel S, Ehrentraut S, Tomasch J, Lienenklaus S, Schneider B, et al. (2012) Transcriptional activation of prostate specific homeobox gene NKX3-1 in subsets of T-cell lymphoblastic leukemia (T-ALL). PLoS One. 7(7): e40747.

15. Soulier J, Clappier E, Cayuela JM, Regnault A, García-Peydró M, et al. (2005) HOXA genes are included in genetic and biologic networks defining human acute T-cell leukemia (T-ALL). Blood. 106(1): 274-286.

16. Marschalek R (2011) Mechanisms of leukemogenesis by MLL fusion proteins. Br J Haematol. 152(2): 141-154.

17. Slany RK (2009) The molecular biology of mixed lineage leukemia. Haematologica. 94(7): 984-993.

18. Drexler HG (2010) Guide to Leukemia-Lymphoma Cell Lines., $2^{\text {nd }}$ edition, Braunschweig, (compact disc).

19. Nagel S, Venturini L, Marquez VE, Meyer C, Kaufmann M, et al. (2010) Polycomb repressor complex 2 regulates HOXA9 and HOXA10, activating ID2 in NK/T-cell lines. Mol Cancer 9: 151.

20. Lenz G, Wright GW, Emre NC, Kohlhammer H, Dave SS, et al. (2008) Molecular subtypes of diffuse large B-cell lymphoma arise by distinct genetic pathways. Proc Natl Acad Sci U S A. 105(36): 13520-13525.

21. MacLeod RA, Kaufmann M, Drexler HG (2007) Cytogenetic harvesting of commonly used tumor cell lines. Nat Protoc. 2(2): 372-382.

22. MacLeod RA, Kaufmann M, Drexler HG (2011) Cytogenetic analysis of cancer cell lines. Methods Mol Biol. 731: 57-78

23. Nagel S, Burek C, Venturini L, Scherr M, Quentmeier H, et al. (2007) Comprehensive analysis of homeobox genes in Hodgkin lymphoma cell lines identifies dysregulated expression of HOXB9 mediated via ERK5 signaling and BMI1. Blood 109(7): 3015-3023.

24. Quentmeier H, Eberth S, RomaniJ, Weich HA, Zaborski M, et al. (2012) DNA methylation regulates expression of VEGF-R2 (KDR) and VEGF-R3 (FLT4). BMC Cancer 12: 19.

25. Rao V, Guan B, Mutton LN, Bieberich CJ (2012) Proline-mediated Proteasomal Degradation of the Prostate-specific Tumor Suppressor NKX3.1. J Biol Chem. 287(43): 36331-36340

26. Heisig J, Weber D, Englberger E, Winkler A, Kneitz S, et al. (2012) Target gene analysis by microarrays and chromatin immunoprecipitation identifies HEY proteins as highly redundant bHLH repressors. PLoS Genet. 8(5): e1002728.

27. Nagel S, Schneider B, Meyer C, Kaufmann M, Drexler HG, et al. (2012) Transcriptional deregulation of homeobox gene ZHX2 in Hodgkin lymphoma. Leuk Res. 36(5): 646-655.

28. Nagel S, Venturini L, Przybylski GK, Grabarczyk P, Meyer C, et al. (2009) NKlike homeodomain proteins activate NOTCH3-signaling in leukemic T-cells. BMC Cancer 9: 371.

29. Bernstein BE, Mikkelsen TS, Xie X, Kamal M, Huebert DJ, et al. (2006) A bivalent chromatin structure marks key developmental genes in embryonic stem cells. Cell 125(2): 315-326.

30. Wang J, Muntean AG, Hess JL (2012) ECSASB2 mediates MLL degradation during hematopoietic differentiation. Blood 119(5): 1151-1161.

31. Hess JL (2004) MLL: a histone methyltransferase disrupted in leukemia. Trends Mol Med. 10(10): 500-507.

32. Lee JS, Shukla A, Schneider J, Swanson SK, Washburn MP, et al. (2007) Histone crosstalk between H2B monoubiquitination and $\mathrm{H} 3$ methylation mediated by COMPASS. Cell 131(6): 1084-1096.

33. Fuchs G, Shema E, Vesterman R, Kotler E, Wolchinsky Z, et al. (2012) RNF20 and USP44 regulate stem cell differentiation by modulating H2B monoubiquitylation. Mol Cell 46(5): 662-673.

34. Margueron R, Reinberg D (2011) The Polycomb complex PRC2 and its mark in life. Nature 469(7330): 343-349.

35. Mosammaparast N, Shi Y (2010) Reversal of histone methylation: biochemical and molecular mechanisms of histone demethylases. Annu Rev Biochem. 79: $155-179$.

36. Kee BL, Rivera RR, Murre C (2001) Id3 inhibits B lymphocyte progenitor growth and survival in response to TGF-beta. Nat Immunol. 2(3): 242-247.

37. Li C, Zhu NL, Tan RC, Ballard PL, Derynck R, et al. (2002) Transforming growth factor-beta inhibits pulmonary surfactant protein $\mathrm{B}$ gene transcription through SMAD3 interactions with NKX2.1 and HNF-3 transcription factors. J Biol Chem. 277(41): 38399-38408.
38. Homminga I, Pieters R, Langerak AW, de Rooi JJ, Stubbs A, et al. (2011) Integrated transcript and genome analyses reveal NKX2-1 and MEF2C as potential oncogenes in T cell acute lymphoblastic leukemia. Cancer Cell 19(4): $484-497$.

39. Garraway LA, Sellers WR (2006) Lineage dependency and lineage-survival oncogenes in human cancer. Nat Rev Cancer 6(8): 593-602.

40. Kwei KA, Kim YH, Girard L, Kao J, Pacyna-Gengelbach M, et al. (2008) Genomic profiling identifies TITF1 as a lineage-specific oncogene amplified in lung cancer. Oncogene 27(25): 3635-3640.

41. Kendall J, Liu Q, Bakleh A, Krasnitz A, Nguyen KC, et al. (2007) Oncogenic cooperation and coamplification of developmental transcription factor genes in lung cancer. Proc Natl Acad Sci USA. 104(42): 16663-16668.

42. Watanabe H, Francis JM, Woo MS, Etemad B, Lin W, et al. (2013) Integrated cistromic and expression analysis of amplified NKX2-1 in lung adenocarcinoma identifies LMO3 as a functional transcriptional target. Genes Dev. 27(2): 197210.

43. Massari ME, Murre C (2000) Helix-loop-helix proteins: regulators of transcription in eucaryotic organisms. Mol Cell Biol. 20(2): 429-440.

44. O'Neil J, Shank J, Cusson N, Murre C, Kelliher M (2004) TAL1/SCL induces leukemia by inhibiting the transcriptional activity of E47/HEB. Cancer Cell 5(6): 587-596.

45. Köchert K, Ullrich K, Kreher S, Aster JC, Kitagawa M, et al. (2011) High-level expression of Mastermind-like 2 contributes to aberrant activation of the NOTCH signaling pathway in human lymphomas. Oncogene 30(15): 18311840.

46. Favre CJ, Mancuso M, Maas K, McLean JW, Baluk P, et al. (2003) Expression of genes involved in vascular development and angiogenesis in endothelial cells of adult lung. Am J Physiol Heart Circ Physiol. 285(5): H1917-1938.

47. Deisch J, Fuda FB, Chen W, Karandikar N, Arbini AA, et al. (2009) Segmental tandem triplication of the MLL gene in an intravascular large B-cell lymphoma with multisystem involvement: a comprehensive morphologic, immunophenotypic, cytogenetic, and molecular cytogenetic antemortem study. Arch Pathol Lab Med. 133(9): 1477-1482.

48. Shema E, Tirosh I, Aylon Y, Huang J, Ye C, et al. (2008) The histone H2Bspecific ubiquitin ligase RNF20/hBRE1 acts as a putative tumor suppressor through selective regulation of gene expression. Genes Dev. 22(19): 2664-2676.

49. Attwooll C, Oddi S, Cartwright P, Prosperini E, Agger K, et al. (2005) A novel repressive E2F6 complex containing the polycomb group protein, EPC1, that interacts with EZH2 in a proliferation-specific manner. J Biol Chem. 280(2): $1199-1208$

50. Kee HJ, Kim JR, Nam KI, Park HY, Shin S, et al. (2007) Enhancer of polycomb1, a novel homeodomain only protein-binding partner, induces skeletal muscle differentiation. J Biol Chem. 282(10): 7700-7709.

51. Chen Y, Pacyna-Gengelbach M, Deutschmann N, Niesporek S, Petersen I (2007) Homeobox gene HOP has a potential tumor suppressive activity in human lung cancer. Int J Cancer 121(5): 1021-1027.

52. Sánchez-Beato M, Sánchez E, García JF, Pérez-Rosado A, Montoya MC, et al. (2004) Abnormal PcG protein expression in Hodgkin's lymphoma. Relation with E2F6 and NFkappaB transcription factors. J Pathol. 204(5): 528-537.

53. Spitz F, Furlong EE (2012) Transcription factors: from enhancer binding to developmental control. Nat Rev Genet. 13(9): 613-626.

54. Nagel S, Schneider B, Rosenwald A, Meyer C, Kaufmann M, et al. (2011) $\mathrm{t}(4 ; 8)(\mathrm{q} 27 ; \mathrm{q} 24)$ in Hodgkin lymphoma cells targets phosphodiesterase PDE5A and homeobox gene ZHX2. Genes Chromosomes Cancer 50(12): 996-1009.

55. Vierbuchen T, Wernig M (2012) Molecular roadblocks for cellular reprogramming. Mol Cell 47(6): 827-838.

56. Bussmann LH, Schubert A, Vu Manh TP, De Andres L, Desbordes SC, et al. (2009) A robust and highly efficient immune cell reprogramming system. Cell Stem Cell 5(5): 554-566.

57. Kulessa H, Frampton J, Graf T (1995) GATA-1 reprograms avian myelomonocytic cell lines into eosinophils, thromboblasts, and erythroblasts. Genes Dev. 9(10): 1250-1262.

58. Antonica F, Kasprzyk DF, Opitz R, Iacovino M, Liao XH, et al. (2012) Generation of functional thyroid from embryonic stem cells. Nature 491: 66-71.

59. Palii CG, Perez-Iratxeta C, Yao Z, Cao Y, Dai F, et al. (2011) Differential genomic targeting of the transcription factor TAL1 in alternate haematopoietic lineages. EMBO J. 30(3): 494-509.

60. Xu J, Shao Z, Glass K, Bauer DE, Pinello L, et al. (2012) Combinatorial assembly of developmental stage-specific enhancers controls gene expression programs during human erythropoiesis. Dev Cell 23(4): 796-811.

61. Kubicek S, O’Sullivan RJ, August EM, Hickey ER, Zhang Q et al. (2007) Reversal of H3K9me2 by a small-molecule inhibitor for the G9a histone methyltransferase. Mol Cell 25(3): 473-481. 